\title{
Full instability behavior of $N$-dimensional dynamical systems with a one-directional nonlinear vector field
}

\author{
J. Rius, ${ }^{1}$ M. Figueras,${ }^{1}$ R. Herrero, ${ }^{1}$ F. Pi, ${ }^{1}$ J. Farjas,${ }^{1,2}$ and G. Orriols ${ }^{1}$ \\ ${ }^{1}$ Departament de Física, Universitat Autònoma de Barcelona, 08193 Bellaterra, Spain \\ ${ }^{2}$ Departament de Física, Universitat de Girona, Avinguda Santaló, s/n 17071 Girona, Spain
}

(Received 21 September 1999; revised manuscript received 11 February 2000)

\begin{abstract}
We show how certain $\mathrm{N}$-dimensional dynamical systems are able to exploit the full instability capabilities of their fixed points to do Hopf bifurcations and how such a behavior produces complex time evolutions based on the nonlinear combination of the oscillation modes that emerged from these bifurcations. For really different oscillation frequencies, the evolutions describe robust wave form structures, usually periodic, in which selfsimilarity with respect to both the time scale and system dimension is clearly appreciated. For closer frequencies, the evolution signals usually appear irregular but are still based on the repetition of complex wave form structures. The study is developed by considering vector fields with a scalar-valued nonlinear function of a single variable that is a linear combination of the $N$ dynamical variables. In this case, the linear stability analysis can be used to design $N$-dimensional systems in which the fixed points of a saddle-node pair experience up to $N-1$ Hopf bifurcations with preselected oscillation frequencies. The secondary processes occurring in the phase region where the variety of limit cycles appear may be rather complex and difficult to characterize, but they produce the nonlinear mixing of oscillation modes with relatively generic features.
\end{abstract}

PACS number(s): 05.45.- a, 42.65.Pc

\section{INTRODUCTION}

Complexity in nonlinear dynamics appears typically associated with the irregularity of chaos. A dissipative system evolving in a chaotic state describes a recurrent motion based on a few basic trajectories that, appearing somewhat different at each turn, follow a definite sequence without apparent regular order and very sensitive to small changes of both variables and parameters $[1,2]$. Usually, however, the basic trajectories are of simple structure and involve a very low number of characteristic frequencies.

A different picture was contained in the physical mechanism tentatively proposed by Landau to explain the initiation of turbulence in fluids [3]. The process is based on a sequence of oscillatory instabilities that, starting from the stationary laminar flow, would produce quasiperiodic evolutions of complex structure due to the large number of different characteristic frequencies. The role of the nonlinearities in this analysis is just to stabilize the oscillatory motion that emerges, from each instability, while the successive oscillation modes combine in a direct manner and with arbitrary relative phases. In light of the bifurcation theory of dynamical systems, the Landau sequence may be interpreted as the Hopf bifurcation of a fixed point followed by secondary bifurcations generating invariant tori of successively higher dimension. This sequence of bifurcations is not considered a route to chaos because small perturbations of triply periodic flows on three-dimensional tori can already yield strange attractors [4], but it remains a way to incorporate additional degrees of freedom in the oscillation dynamics of high-dimensional systems.

In this work we show the emergence of complex dynamical behavior through the nonlinear oscillatory superposition of different characteristic frequencies generated by Hopf bifurcations. In relation to the Landau scenario, our problem is simpler because it deals with systems of finite dimension based on vector fields with a uniformly directed nonlinear part, i.e., $\mathrm{N}$-dimensional vector fields containing a single scalar-valued nonlinear function. Nevertheless, while the Landau scenario develops from a single node point and a single sequence of torus bifurcations, we consider more than one fixed point because the nonlinear mechanisms can mix the oscillatory dynamics emerged from neighboring points. And, on the other hand, we investigate situations where the fixed points experience successive Hopf bifurcations and a variety of limit cycles emerge in phase space. Finally, in relation to the Landau proposal, a dynamical system cannot exhibit arbitrary phase relationships between different oscillation modes, but the superposition takes place nonlinearly and the mode mixing processes may be responsible for rather complex wave forms in the time dynamics.

For vector fields with a one-directional nonlinear part, the fixed points appear aligned in an alternate sequence of saddle-node type and, typically, the observed dynamics is associated with an attractor arising from one of the nodes and growing under the influence of the nearest saddle point. We will show how certain $\mathrm{N}$-dimensional systems of this type are able to exploit all the instability capabilities of a saddlenode pair of fixed points by experiencing a total of $N-1$ Hopf bifurcations on them [5]. The points initially have stable manifolds of dimension $N-1$ and $N$, respectively, and after the $N-1$ Hopf bifurcations one of them has become fully unstable while the other possesses only one stable dimension. A variety of limit cycles have emerged from the points and some invariant tori could also have been created through secondary Hopf bifurcations of the cycles. The majority of such limit sets are saddles, and a few number of attractors [6] live in the middle of the intertwinement of saddle outstructures. The time evolution associated with one of the attractors can manifest the influence of neighboring saddles and, under such circumstances, the most prominent 

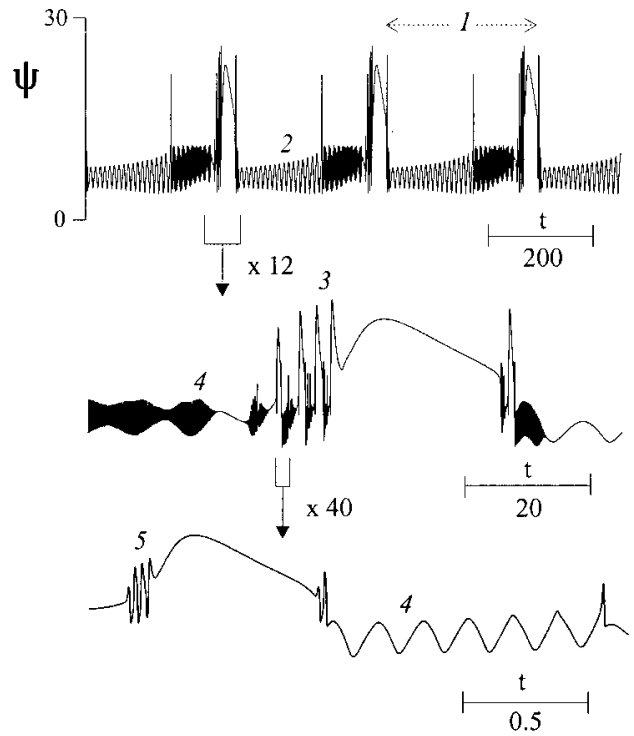

FIG. 1. Time evolution illustrating the full instability behavior of a six-dimensional system in which a saddle-node pair of fixed points have experienced two and three Hopf bifurcations, respectively. Notice the presence of oscillations at five characteristic frequencies, $\omega_{q}$, which, ordered from low to high, are identified in the signal by the number $q$. The nonlinear function is the Gaussian given by Eq. (A1), and the other parameters are $\mu_{c}=16, \omega_{H q}$ $=0.1,0.7,4,35,250, p_{H q}=-0.7,3,-1,3.2,-2.2$, and $c_{1}$ $=600=2.4 \omega_{H 5}$.

$\mathrm{N}$-dimensional feature to be observed is the appearance of $N-1$ oscillation modes nonlinearly combined in the time dynamics. This is what we call the full instability behavior of the $N$-dimensional dynamical system, and Fig. 1 presents a numerical example of such a behavior for $N=6$. In this case, the various Hopf bifurcations have generated really different oscillation frequencies and the time signal shows a wave form structure with clear distinction of the $N-1$ modes. This figure is commented on in Sec. V, together with other numerical simulations, and we now want only to remark on both the complexity and robustness of the wave form structure. In this case, the signal is periodic, and it is therefore evident that the wave form complexity is independent of chaos.

The first step for investigating the full instability behavior is to dispose of systems able to exhaust the stable dimensions of their fixed points through successive Hopf bifurcations. The participation of all the dynamical variables within the nonlinearities clearly seems necessary, but the influence of the nonlinear functional structure is difficult to predict. With this purpose in mind, we have developed a method of linear stability analysis for studying the bifurcation possibilities of a set of $N$-dimensional fixed points in as general a way as possible. The analysis is not performed in the parameter space, where it may in fact be impossible, but we consider the space defined by the coefficients of the characteristic equation. Every point of that space can be associated with the corresponding set of $N$ eigenvalues, and the linear stability analysis of a given system can be performed by locating its fixed points in the same space. The simplest and most manageable situation corresponds to systems whose fixed points appear located on a straight line and move on that line under variation of a proper parameter. This happens for systems based on a scalar-valued nonlinear function of a single variable when that variable is a linear combination of the dynamic variables and when the control parameter is a scale factor on the nonlinear function. In this case it is possible to design the dynamical systems in order to obtain the occurrence of $N-1$ Hopf bifurcations on a saddle-node pair of fixed points, with a prefixed set of values for the oscillation frequencies and within a prefixed range of the control parameter. The numerical examples reported in the paper correspond to this situation.

In addition to the Hopf bifurcations of the fixed points, the full instability behavior requires mode mixing mechanisms yielding the complex wave forms. In the case of nonlinear functions of a single variable that is a linear combination of the $N$ dynamical variables, the mode mixing seems strongly related to the Hopf bifurcations and, if the fixed points have done or are near to doing the $N-1$ Hopf bifurcations, the system then exhibits a signal of the type shown in Fig. 1. The secondary processes may be rather complex and dependent on the circumstances, but they do not introduce additional characteristic frequencies, and the nonlinear mode mixing produces time evolutions with qualitatively generic features.

It is worth emphasizing that this work has been motivated by a family of physical systems, the so-called BOITAL devices [7], whose effective dynamical dimension can be easily varied and who are able to exhibit the full instability behavior in a natural way. Experimental results obtained with BOITAL devices of up to $N=6$ will be reported elsewhere [8], and some of the numerical examples reported here are based on the mathematical model developed for describing such devices $[9,10]$.

\section{II. $N$-DIMENSIONAL SYSTEMS WITH A ONE-DIRECTIONAL NONLINEAR VECTOR FIELD}

We consider systems based on $N$-dimensional vector fields whose nonlinear part has a uniform direction across the full phase space and that may be written as

$$
\dot{z}=A z+b f(z ; \mu)
$$

where $z(t)$ represents the vector state, $f(z, \mu)$ is a scalarvalued function nonlinear on $z, \mu$ describes the set of parameters involved in the nonlinear function, $A$ is a constant $N$ $\times N$ matrix, and $b$ is a constant vector defining the direction of the nonlinear component of the field. A variety of well known low-dimensional dynamical systems are based on a single scalar-valued nonlinear function: the Duffing [11] and van der Pol [12] oscillators, the Lotka-Volterra model [13], the Rössler model [14], and the Chua circuit and oscillator [15].

The equilibria of the system (1) appear in phase space located on a straight line determined by $A$ and $b$. It suggests a coordinate transformation so that the line of fixed points is one of the new axes. Particularly useful is the transformation of system (1) into the canonical form based on the companion matrix [16] as follows: 


$$
\begin{gathered}
\dot{x}_{1}=-\sum_{j=1}^{N} c_{j} x_{j}+f\left(x_{1}, \ldots, x_{N} ; \mu\right), \\
\dot{x}_{j}=x_{j-1}, \quad j=2, \ldots, N,
\end{gathered}
$$

which, with the definition $x_{N}=y$, can be written in the classical form

$$
\begin{aligned}
y^{(N)} & +c_{1} y^{(N-1)}+\ldots+c_{N-1} y^{(1)}+c_{N} y \\
= & f\left(y^{(N-1)}, \ldots, y^{(1)}, y ; \mu\right),
\end{aligned}
$$

where the superscripts denote the order of differentiation with respect to time. Notice that in this representation the nonlinear part of the vector field is directed along the $x_{1}$ or $y^{(N-1)}$ coordinate while the equilibrium points appear located on the $x_{N}$ or $y$ axis.

We now introduce an explicit control parameter by assuming that the nonlinear function includes a scale factor as follows:

$$
f\left(x_{1}, \ldots, x_{N} ; \mu\right)=\mu_{c} g\left(x_{1}, \ldots, x_{N} ; \mu\right),
$$

with $\mu_{c}$ supposed to be independent of the $c_{j}$ coefficients. Although such a kind of parameter cannot be available in a given application, it is really useful for analytic purposes because it simply modifies the relative weight of the nonlinear and linear components of the vector field without altering their structure. This property will allow us to consider the linear stability of the steady state bifurcation diagram as a function of $\mu_{c}$ without particular specifications about the nonlinear function, other than the assumption that it is continuous and differentiable.

On the other hand, the full instability behavior probably requires the nonlinear participation of all the dynamic variables and the simplest situation is that in which the nonlinear function affects a linear combination of the $N$ variables. Thus we consider nonlinear functions of a single variable as follows:

$$
f\left(x_{1}, \ldots, x_{N} ; \mu\right)=f(\psi ; \mu),
$$

where

$$
\psi=\sum_{1}^{N} d_{j} x_{j}
$$

Notice that the coefficients $d_{j}$ can be transported to the linear part of the vector field by defining the $d_{j} x_{j}$ as a new set of variables. The roles of the linear and nonlinear parts of the vector field appear well differentiated with this kind of function. In addition, it permits one to adjust the relative participation of the several variables to the feedback loop independently of the nonlinear function itself. The Lotka-Volterra model [13], the Duffing [11] and van der Pol [12] oscillators, the Chua circuit [15], and the BOITAL devices [7] are systems with a nonlinear function of a single variable in the form $(5 b)$.

\section{STEADY-STATE SOLUTION} by

The steady-state solution of Eqs. (2) and (4) is determined

$$
\begin{gathered}
\bar{x}_{j}=0, \quad j=1, \ldots, N-1, \\
c_{N} \bar{x}_{N}=\mu_{c} G\left(\bar{x}_{N} ; \mu\right),
\end{gathered}
$$

where the overline denotes steady state values and $G\left(x_{N} ; \mu\right)=g\left(x_{j \neq N}=0, x_{N} ; \mu\right)$. Thus the number of fixed points and their positions on the $x_{N}$ axis depend only on the nonlinearities exclusively involving $x_{N}$. For a linear combination of variables like that in Eq. (5b), the functions $g$ and $G$ are equivalent and Eq. (7) may be written as follows:

$$
\bar{\psi}=d_{N} \bar{x}_{N}=\frac{\mu_{c} d_{N}}{c_{N}} g(\bar{\psi}) .
$$

This condition may be graphically analyzed, as shown in Fig. 2 for the nonlinear functions used in the numerical simulations. These functions are described in Appendix A, and here we illustrate generic features of the steady-state solution relevant for the method of linear stability analysis. In the lefthand column of Fig. 2, each function $g(\psi)$ is represented together with the straight line $g(\psi)=\left(c_{N} / \mu_{c} d_{N}\right) \psi$. The intersections of this line with the nonlinear function determine the solutions $\bar{\psi}$ for the considered value of $\mu_{c}$, and by changing the line slope one obtains the steady state bifurcation diagram as a function of $\mu_{c}$ (middle column of Fig. 2). For a bounded nonlinear function, a single solution exists for $\mu_{c}=0$, and additional solutions appear with increasing $\mu_{c}$ every time the straight line becomes tangent to the nonlinear function. For a continuous function, the branching diagram as a function of $\mu_{c}$ appears continuously connected without isolas.

We will see in Sec. IV that the ratio between the slopes of the nonlinear function and the straight line at their intersection is useful for characterizing the linear stability of the steady-state solution. The ratio of slopes is defined as the value

$$
p(\bar{\psi})=\frac{d_{N}}{c_{N}} \mu_{c}\left[\frac{\partial g}{\partial \psi}\right]_{\bar{\psi}}=\frac{\bar{\psi}}{g(\bar{\psi})}\left[\frac{\partial g}{\partial \psi}\right]_{\bar{\psi}},
$$

and the right-hand column of Fig. 2 presents the distribution of $p$ values upon the branching diagrams. The branches represented by the solid (dashed) line correspond to $p<1$ ( $p$ $>1)$ and the zero eigenvalue bifurcations occur at the $p$ $=1$ connections.

If the control parameter is rescaled to $\mu_{c} d_{N} / c_{N}[17]$, then it results that all of the systems based on a given nonlinear function $g(\psi)$, but having arbitrary dimension $N$ and arbitrary values for the $c_{j}$ and $d_{j}$ coefficients, are associated with the same steady state branching diagram describing $\bar{\psi}$ versus $\mu_{c} d_{N} / c_{N}$. In addition, the distribution of $p$ values upon this branching diagram is the same for all of these systems. This is a particularly useful situation for analyzing systems of gradually increasing dimension. 

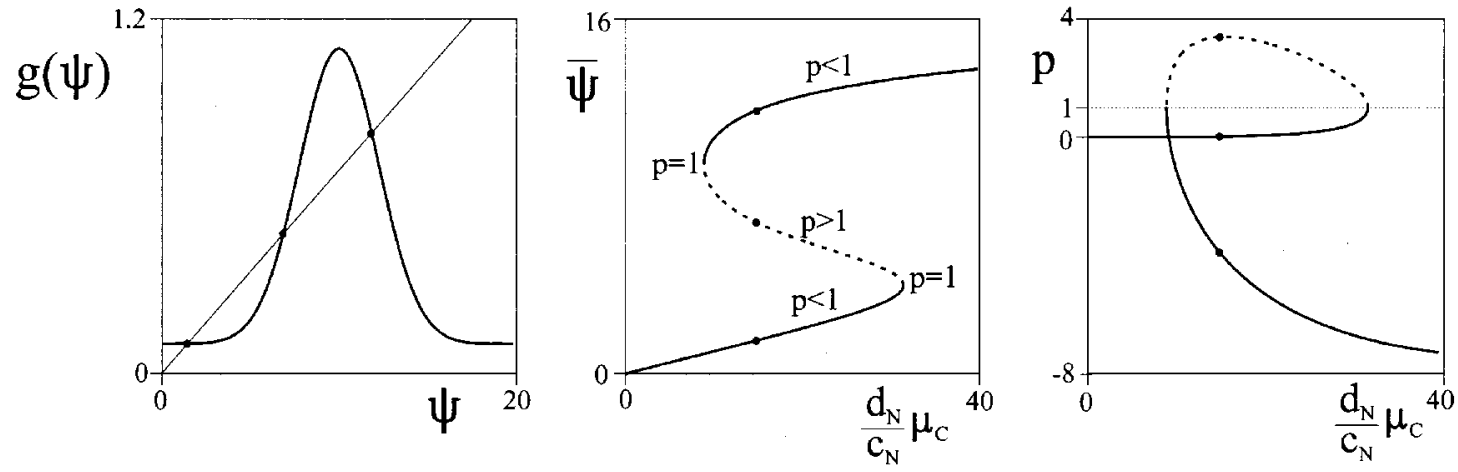

(a)
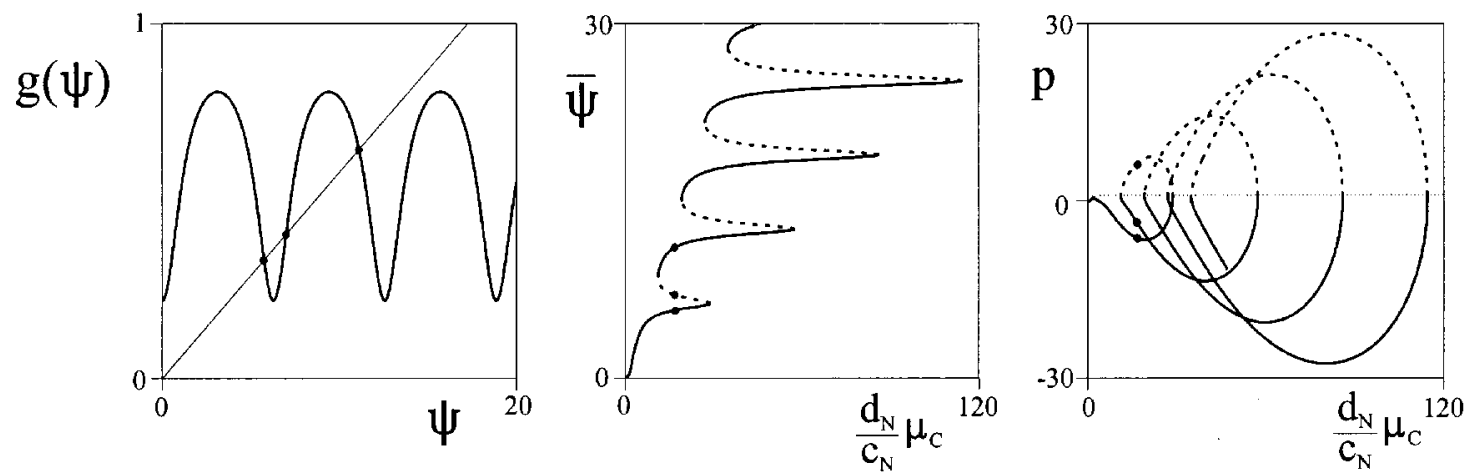

(b)
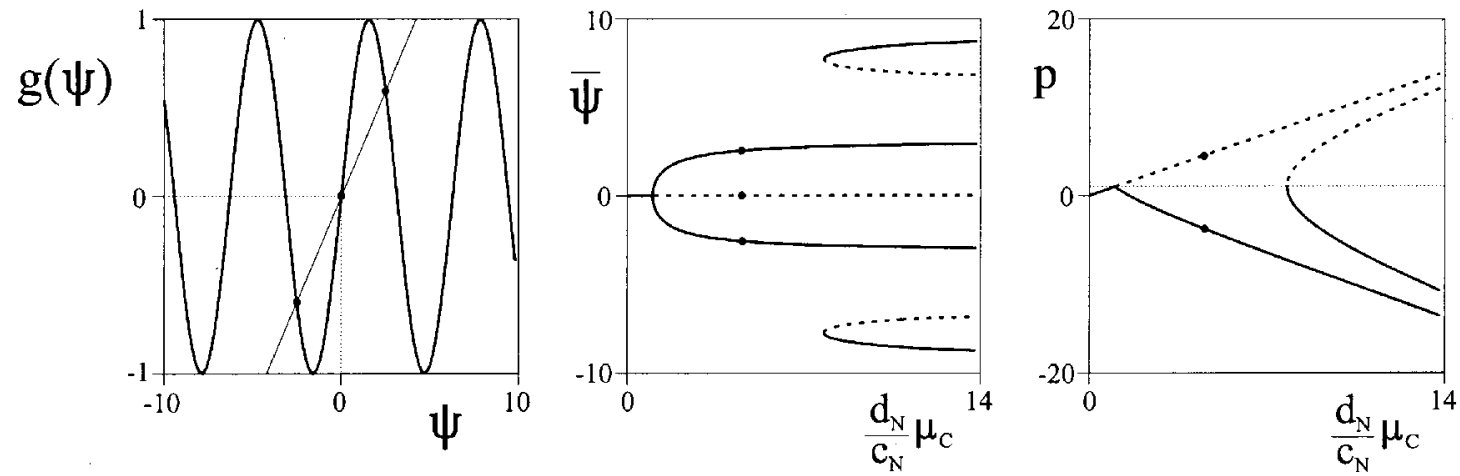

(c)

FIG. 2. Nonlinear functions used in the numerical simulations, accompanied by the corresponding steady-state branching diagrams and $p$-value distributions. The straight line represented over each nonlinear function is given by Eq. (8) and their intersections determine the steady-state solutions for a given $\mu_{c}$ value.

\section{LINEAR STABILITY ANALYSIS}

The Jacobian matrix of Eq. (2) is in the companion form, and the characteristic equation has then the coefficients directly given by the Jacobian elements. The coefficients associated with a given fixed point are

$$
k_{j}(\bar{x})=c_{j}-\left[\frac{\partial f}{\partial x_{j}}\right]_{\bar{x}}, \quad j=1, \ldots, N .
$$

The simplicity of these relations allows us to develop an unconventional representation of the linear stability analysis. Instead of the parameter space, we consider the intermediate space defined by the coefficients $k_{j}$. Every point of this space may be associated with the corresponding set of $N$ eigenvalues determined by the characteristic equation. This is a universal representation describing the full variety of linearized $N$-dimensional fixed points, in which the nonhyperbolic points and eigenvalue degeneracies are easily located. On the other hand, for systems in the canonical form
(2), Eqs. (10) identify the position of the fixed points in the $k_{j}$ space and in this way we can know their stability. The variation of a parameter moves the fixed points on certain curves and their intersections with the loci of nonhyperbolic points denote the steady-state bifurcations. In particular, for nonlinear functions with a control parameter like $\mu_{c}$ in Eq. (4), the continuity of the steady-state solution connects the motions of the several fixed points in a single curve, and this permits one to associate the $\mu_{c}$-parameter family of systems with that curve in the $k_{j}$ space.

The method is really useful for systems with a nonlinear function of a single variable in the form (5b) because in this case Eqs. (10) may be written as

$$
k_{j}(\bar{\psi})=c_{j}-\mu_{c}\left[\frac{\partial g}{\partial \psi}\right]_{\bar{\psi}} d_{j}=c_{j}-p \frac{c_{N}}{d_{N}} d_{j}, \quad j=1, \ldots, N,
$$

with $p$ given by Eq. (9). Thus the fixed points of a $\mu_{c}$-parameter family of systems move on the straight line 
passing for the point of coordinates $\left(c_{j}\right)$ with a director vector determined by the set of coefficients $d_{j}$. For $d_{N}$ and $c_{N}$ $\neq 0$, the $p$ value of a fixed point determines its position on the line and therefore determines its linear stability behavior. On the other hand, Eq. (10) for $j=N$,

$$
k_{N}(\bar{\psi})=c_{N}(1-p)
$$

gives a useful relation between the $k_{N}$ coordinates and $p$ values of the fixed points. Recall that the steady state solution as a function of $\mu_{c}$ always appears organized in branches with either $p<1$ or $p>1$ and with the branch connections at $p=1$ (see Fig. 2). Equation (12) locates the two kinds of branches on opposite sides of the subspace $k_{N}=0$.

The linear stability of the steady-state solution may be easily characterized by using $p$ instead of $\mu_{c}$ for relating the branching bifurcation diagram with the straight line of the $\mu_{c}$ family of systems in the $k_{j}$ space. The most significant $p$ values are those of the intersections between the line and the surfaces of nonhyperbolic points. The intersection with the $k_{N}=0$ subspace corresponds to $p=1$ and describes the zero eigenvalue bifurcations. The intersections with the locus of the $\lambda_{ \pm}= \pm i \omega$ nonhyperbolic points denote the occurrence of Hopf bifurcations and the corresponding set of $p$ values may be used to locate these bifurcations on the branching diagram.

In summary, the systems with a one-directional nonlinear field of a single variable in the form (5b) and a control parameter like that in Eq. (4) have two peculiar features:

(i) The nonlinear function $g(\psi)$ determines both the steady-state branching diagram describing $\bar{\psi}$ as a function of $\mu_{c}$ and the distribution of $p$ values upon that diagram, independently of $N, c_{j}$, and $d_{j}$.

(ii) The coefficients $c_{j}$ and $d_{j}$ determine the straight line of Eqs. (11) in the $N$-dimensional $k_{j}$ space. The position of this line with respect to the surfaces of nonhyperbolic points delimits the linear stability behavior of the $\mu_{c}$ family of systems, independently of $g(\psi)$. The fixed points appear to be located on the straight line according to their actual $p$ values and can therefore be moved on that line by varying $\mu_{c}$ or $g(\psi)$.

\section{A. Loci of nonhyperbolic points in the $k_{j}$ space}

The points of the $k_{j}$ space can be classified according to the number of eigenvalues with positive real parts, and this means $N+1$ classes of points having from 0 to $N$ unstable dimensions. Since the eigenvalues vary continuously, the space appears organized in $N+1$ different regions delimited by the loci of nonhyperbolic points. The nonhyperbolic points are of the types $\left\{0^{m}\right\},\left\{ \pm i \omega_{1}, \ldots, \pm i \omega_{m}\right\}$, or $\left\{0^{n}\right.$, $\left.\pm i \omega_{1}, \ldots, \pm i \omega_{m-n}\right\}$, where $0^{m}$ denotes $m$ real eigenvalues equal to 0 and $\pm i \omega_{j}$ denotes a complex conjugated pair with zero real part and arbitrary imaginary part. The locus of nonhyperbolic points of a given type is a surface of dimension $N-m$, where the codimension $m$ is the number of independent eigenvalues with zero real part in the nonhyperbolic point.

The surfaces emerge from the codimension- $N$ point at the origin of the $k_{j}$ space, and their intersection with any hypersphere enclosing that point produces qualitatively equivalent structures. This self-similar property works in accordance with the invariance of the characteristic equation to time rescaling. The surface of a given type of nonhyperbolic points will be denoted like the points, and it may be seen that

(i) $\left\{0^{m}\right\}$ is the linear subspace of dimension $N-m$ defined by

$$
k_{N}=k_{N-1}=\cdots=k_{N-m+1}=0 .
$$

In particular, $\left\{0^{N}\right\}$ is the origin and $\{0\}$ is the subspace of dimension $N-1$ defined by $k_{N}=0$. The rest of the eigenvalues for the points of $\left\{0^{m}\right\}$ are determined by the characteristic equation of order $N-m$. Thus the spatial structure within $\left\{0^{m}\right\}$ is identical to that of the problem of dimension $N-m$ but with the addition of $m$ zero eigenvalues.

(ii) $\{ \pm i \omega\}$ has dimension $N-1$ and is parametrically determined as a function of $\omega$ by either

$$
\begin{gathered}
k_{1}(i \omega)^{N-2}+k_{3}(i \omega)^{N-4}+\cdots+k_{N-3}(i \omega)^{2}+k_{N-1}=0, \\
(i \omega)^{N}+k_{2}(i \omega)^{N-2}+\cdots+k_{N-2}(i \omega)^{2}+k_{N}=0
\end{gathered}
$$

if $N$ is even, or

$$
\begin{aligned}
& k_{1}(i \omega)^{N-1}+k_{3}(i \omega)^{N-3}+\cdots+k_{N-2}(i \omega)^{2}+k_{N}=0 \\
& (i \omega)^{N-1}+k_{2}(i \omega)^{N-3}+\cdots+k_{N-3}(i \omega)^{2}+k_{N-1}=0
\end{aligned}
$$

if $N$ is odd.

The partition of the $k_{j}$ space is done by $\{0\}$ and $\{ \pm i \omega\}$, the two surfaces of dimension $(N-1)$, and by their interconnection in $\left\{0^{2}\right\}$ and $\{0, \pm i \omega\} .\{0\}$ introduces two half spaces, $k_{N}>0$ and $k_{N}<0$, containing regions where the number of unstable eigenvalues is even or odd, respectively. The surface $\{ \pm i \omega\}$ emerges with $\omega=0$ from $\left\{0^{2}\right\}$ and is divided by $\{0, \pm i \omega\}$ in $N-1$ qualitatively different zones where it separates pairs of regions with a different number of unstable dimensions, i.e., $0|2,1| 3,2 \mid 4$, etc. In addition, for $N \geqslant 4$, $\{ \pm i \omega\}$ intersects with itself and yields $\left\{ \pm i \omega_{1}, \pm i \omega_{2}, \ldots\right\}$ degeneracies.

Figure 3 shows the nonhyperbolic surfaces in the $k_{j}$ space for $N=2$ and $N=3$. The case $N=2$ corresponds to the well known two-dimensional linear system and the same structure, with an additional zero eigenvalue, is found in the plane $k_{3}=0$ of the case $N=3$. In its turn, the case $N=3$ also describes the eigenvalue structure of the subspace $\{0\}$ for $N=4$ if a zero eigenvalue is added everywhere.

A more accessible view of the structure of nonhyperbolic surfaces is obtained by considering planes of section of the $k_{j}$ space like those shown in Figs. 4 and 5 for $N=3$ and $N$ $=4$, respectively. The sections are drawn projected on the plane $k_{N-1} k_{N}$ and the numbers denote the unstable dimensions in the different regions. The value of $\omega$ on the surface $\{ \pm i \omega\}$ in the plane of section is also shown. In Fig. 4, both sections cut the $\{0,0\}$ degeneracy, but only the second plane contains the $\{0, \pm i \omega\}$ degeneracy. The appearance of both degeneracies implies the presence of the full variety of regions for $N=3$. In fact, it may be shown that for an arbitrary dimension $N$, a plane can intersect up to $N-1$ times with both the $\{0\}$ and $\{ \pm i \omega\}$ surfaces simultaneously, one time in the $\{0,0\}$ subspace and the rest in the $\{0, \pm i \omega\}$ surface. The full variety of fixed points and the $N-1$ different 


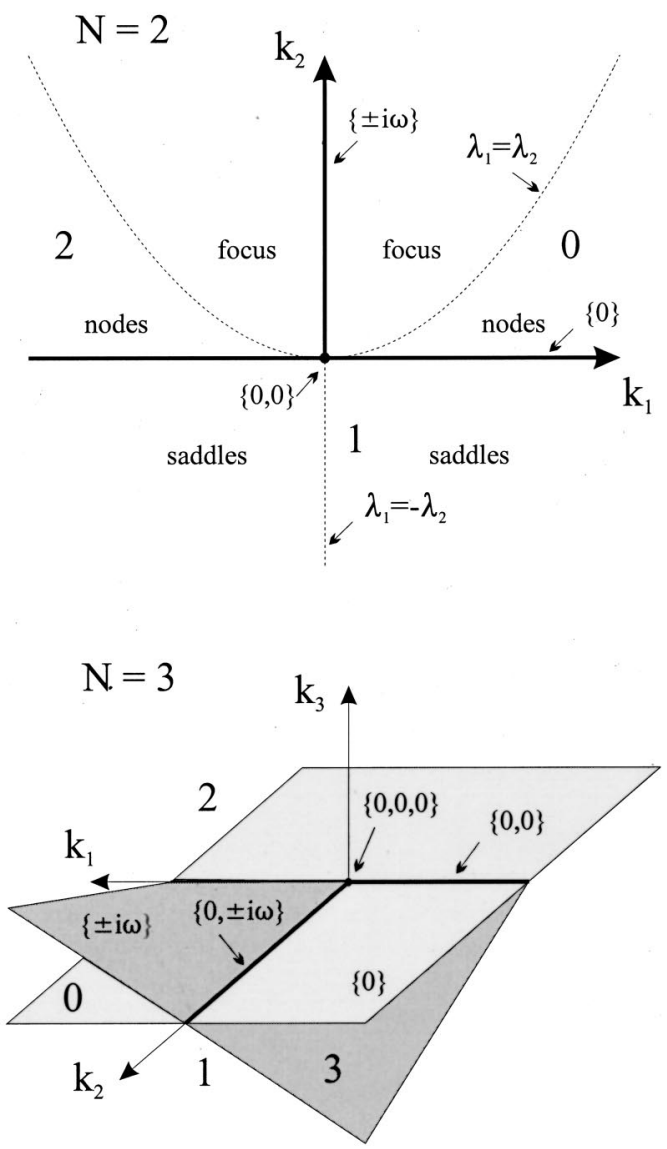

FIG. 3. Loci of nonhyperbolic points in the $k_{j}$ space for $N=2$ and 3 , respectively. Notice the partition in $N+1$ regions with a different number of unstable dimensions.

zones of $\{ \pm i \omega\}$ appear in the plane of section if and only if $N-1$ punctual intersections of $\{0\}$ with $\{ \pm i \omega\}$ also appear. This is what happens in the two cases for $N=4$ of Fig. 5, where the second case contains, in addition, the $\left\{ \pm i \omega_{1}\right.$, $\left.\pm i \omega_{2}\right\}$ degeneracy.

For simplicity, the planes of section represented in Figs. 4 and 5 have been chosen to intersect the surface $\{ \pm i \omega\}$ in a continuous way but, for an arbitrary orientation of the plane, the intersection with that surface will probably present divergences. In any case, it is worth remarking that the surface of a given class of nonhyperbolic points is unique and continuous in the $k_{j}$ space.

\section{B. Eigenvectors for a Jacobian matrix in the companion form}

Unlike what happens with the eigenvalues, the organization of eigenvectors in the $k_{j}$ space is not universal because they depend on the actual Jacobian matrix. Nevertheless, the companion form matrices have the peculiar property that the eigenvectors depend only on the associated eigenvalue as follows:

$$
u_{\lambda}=\left(\lambda^{N-1}, \lambda^{N-2}, \ldots, \lambda, 1\right),
$$

so that the full family of systems in form (2) have the same structure of eigenvectors in the $k_{j}$ space. Notice that the eigenspace associated with an eigenvalue is always onedimensional independently of its multiplicity. (a)
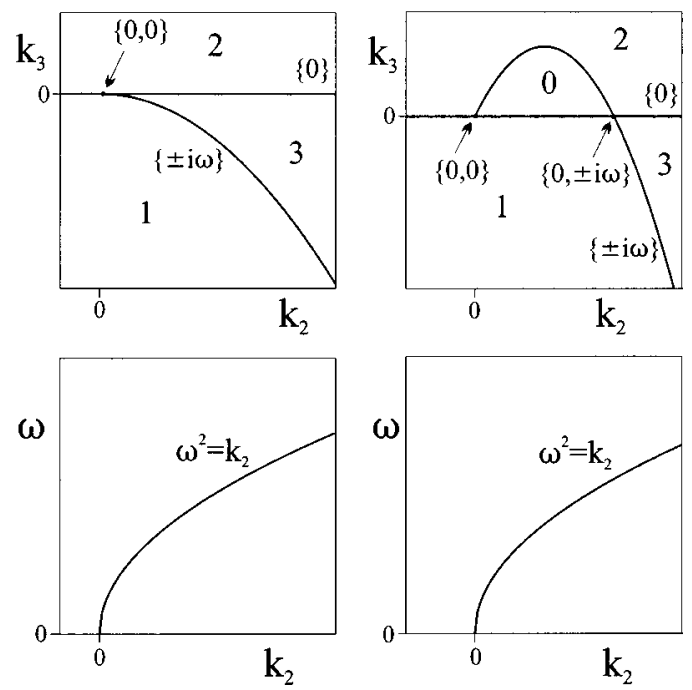

FIG. 4. Surfaces of nonhyperbolic points in two different planes of section of the $k_{j}$ space for $N=3$. Both planes are parallel to $k_{3}$ and cut the other axes with either $k_{1}$ and $k_{2}<0$ or $>0$ in case (a) or (b), respectively. The representations are done projected on the plane $k_{N-1} k_{N}$ and the numerical labels denote the number of unstable dimensions in the different regions. The value of $\omega$ on the surface $\{ \pm i \omega\}$ in the plane of section is also shown.

The eigenvector of the zero eigenvalue is directed along the $x_{N}$ axis everywhere in the subspace $\{0\}$. The twodimensional eigenspace of $\lambda_{ \pm}= \pm i \omega$ depends on the $\omega$ value and is determined by the real vectors

$$
\begin{gathered}
\frac{u_{+}+u_{-}}{2}=\left(\ldots,-\omega^{6}, 0, \omega^{4}, 0,-\omega^{2}, 0,1\right), \\
\frac{u_{+}-u_{-}}{2}=\left(\ldots, \omega^{5}, 0,-\omega^{3}, 0, \omega, 0\right) .
\end{gathered}
$$

(a)
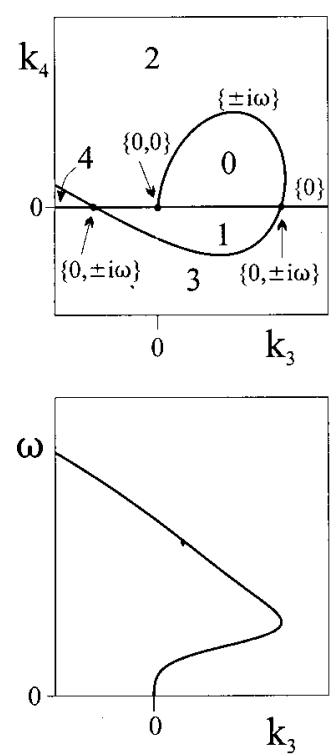

(b)
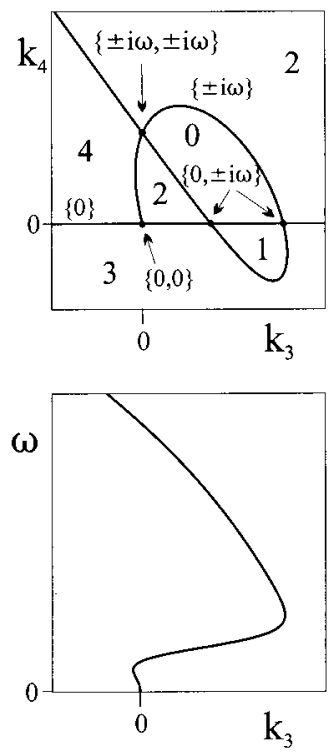

FIG. 5. The same as in Fig. 4 but for $N=4$. 
The value of $\omega$ is equal to zero at the degeneracy $\left\{0^{2}\right\}$ and increases indefinitely when moving away along the surface. Therefore the orientation of the two-dimensional eigenspace changes accordingly.

\section{Fixed points of nonlinear systems in the $\boldsymbol{k}_{j}$ space}

In the presence of nonlinearities, the transverse crossings of the surfaces $\{0\}$ and $\{ \pm i \omega\}$ are usually associated with the saddle-node and Hopf bifurcations, respectively, because the corresponding conditions are generically fulfilled. In a saddle-node bifurcation, a pair of fixed points approach the subspace $k_{N}=0$ from opposite sides, become the same nonhyperbolic point and then disappear (or the opposite process). The saddle-node name is used for convenience but it must be realized that the surface $\{0\}$ appears divided in $N$ parts where the bifurcation involves different kinds of fixed points that are usually a pair of saddles. On the other hand, the crossing of $\{0\}$ can also be associated with either the transcritical or the pitchfork bifurcation, provided the proper conditions are fulfilled, and a different set of fixed points are involved in each case.

In the crossing of $\{ \pm i \omega\}$, a limit cycle would probably emerge around the fixed point. Outside of the center subspace, the limit cycle will maintain the same stability properties as the fixed point. This means that a supercritical Hopf bifurcation occurring between the regions with zero and two unstable dimensions will produce a stable limit cycle, and a subcritical bifurcation between the regions with $N$ and ( $N$ -2 ) unstable dimensions will yield an unstable cycle, but in any other case a kind of saddle cycle will be created. The limit cycles will appear properly oriented in phase space according to the corresponding center subspaces that, in the case of systems in the canonical form (2), depend on the oscillation frequency as expressed by Eqs. (16).

Let us illustrate the method by considering the situation schematically depicted in Fig. 6 for five-dimensional systems in the form (2), (4), and (5). The straight line corresponds to a given set of $c_{j}$ and $d_{j}$ parameters and, in this example, it crosses all the regions containing points with a different number of unstable dimensions. The crossing with $\{0\}$ indicates that any $\lambda=0$ bifurcation will involve fixed points with zero and one unstable dimensions. The crossings with $\{ \pm i \omega\}$ indicate that the fixed points with zero (one) unstable dimensions can suffer successive Hopf bifurcations up to four (five) unstable dimensions and, therefore, it denotes the possibility of achieving the full instability behavior. The nonlinear function $g(\psi)$ determines the number of fixed points and their positions on the line as a function of $\mu_{c}$. The full instability will be achieved if fixed points of the two types, i.e., with $p<1$ and $p>1$, move enough along the line. This may be easily known by comparing the distribution of $p$ values on the steady-state branching diagram with the values $p_{H 1}, \ldots, p_{H 4}$ of the possible Hopf bifurcations, as indicated by arrows in Fig. 6 .

For systems in the form (2) and (4) but with a more general nonlinear function than Eq. (5), the fixed points of a $\mu_{c}$ family will move on a single curve, but now this curve is not straight and can cross the nonhyperbolic surfaces in an arbitrary manner. A more complex motion of fixed points can occur by varying parameters other than $\mu_{c}$. In any case,

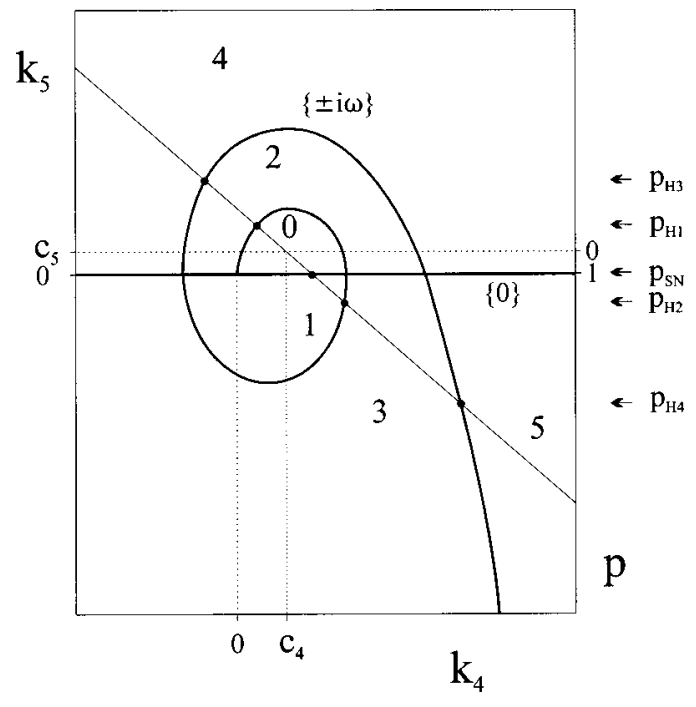

FIG. 6. Schematic example of the method for characterizing the linear stability of five-dimensional systems in the $k_{j}$ space. The straight line describes where the fixed points of the $\mu_{c}$ families of systems in the form (2), (4), and (5) may be for a given set of coefficients $c_{j}$ and $d_{j}$ but for an arbitrary function $g(\psi)$. The righthand vertical scale describes the $p$ value of the fixed points and offers a convenient connection with the steady-state branching diagram. The intersections with the surfaces of nonhyperbolic points indicate the possible steady-state bifurcations.

however, the full instability of the fixed points will be achieved if they reach the regions of highest instability by crossing the surface $\{ \pm i \omega\}$ properly.

\section{Conditions for the full instability}

The situations of full instability can, in principle, be identified by means of the linear stability analysis of the steadystate solution. Nevertheless, in general, it is not easy to establish the corresponding conditions for a given system, and this probably explains why such a behavior has not already been observed. The analysis seems really attainable for systems in the form (2), (4), and (5) because, in this case, it can be divided into two independent problems: one concerning the position and orientation of the straight line with respect to the surface $\{ \pm i \omega\}$, and another dealing with the capabilities of the nonlinear function in producing a steady-state branching diagram with a distribution of $p$ values covering the $p_{H}$ values of the Hopf bifurcations. The latter is easily solved by adjusting $g(\psi)$ properly, but the former is more involved.

From Eqs. (14), it may be seen that the maximum number of intersections between the straight line and $\{ \pm i \omega\}$ is equal to $N-1$. The full instability behavior can be achieved if these intersections occur with the $N-1$ qualitatively different zones of $\{ \pm i \omega\}$, otherwise two of the intersections will correspond to the same Hopf bifurcation but in a contrary direction, and the line will not cross the $N+1$ regions of the $k_{j}$ space. This condition cannot be established in a formal way, and the problem must be indirectly considered, as discussed in Appendix B. Particularly useful is a method for designing the dynamical system, i.e., for determining the coefficients $c_{j}$ and $d_{j}$, in order to achieve the $N-1$ Hopf bifurcations with preselected frequencies and $p_{H}$ values. 


\section{TIME EVOLUTIONS SHOWING THE FULL INSTABILITY BEHAVIOR}

We will now illustrate some features of the full instability behavior when observed in the time evolution of systems in the form (2), (4), and (5) for a variety of nonlinear functions $g(\psi)$, dynamical dimensions $N$, and sets of coefficients $c_{j}$ and $d_{j}$. In each case, the system parameters $c_{j}$ and $d_{j}$ have been defined by preselecting the $\omega_{H}$ and $p_{H}$ values of the $N-1$ Hopf bifurcations and the value of $c_{1}$, as described in Appendix B. The employed nonlinear functions and corresponding steady-state branching diagrams as a function of $\mu_{c}$, as well as the distribution of $p$ values, are presented in Appendix A.

The illustration is done by means of Fig. 1 and the figures of this section. The captions indicate the corresponding nonlinear function and the values of $c_{1}, \omega_{H q}$, and $p_{H q}$. The evolution signals always describe the variable $\psi$ as a function of time for fixed values of $\mu_{c}$ and in general contain a variety of oscillation modes at angular frequencies $\omega_{q}$, which, ordered from lower to higher, are identified on the signals by means of the label $q$.

We first consider the example of Fig. 1 corresponding to a six-dimensional system with a Gaussian nonlinear function, Eq. (A1). The diagrams of Fig. 2(a) show that for $\mu_{c}=16$ the system has three fixed points with $\bar{\psi}=1.6,6.6,12.1$ and $p$ $=0.02,3.4,-4.3$, respectively. The first point remains far from the observed dynamics. The two later points have appeared at $\mu_{c}=9.5$ through a saddle-node bifurcation producing a stable node and a saddle with one unstable dimension. At $\mu_{c}=16$, the node has already become fully unstable after doing successive Hopf bifurcations with frequencies $\omega_{H 1}$, $\omega_{H 3}$, and $\omega_{H 5}$ at the values of $\mu_{c}$ making its $p$ value equal to $p_{H 1}, p_{H 3}$, and $p_{H 5}$, respectively. The first bifurcation, in this case at $\omega_{H 1}$, has produced the stable limit cycle from which the attractor sustaining the time evolution of Fig. 1 is derived. On its turn, the saddle point has also made the two Hopf bifurcations, at $\omega_{H 2}$ and $\omega_{H 4}$, and for $\mu_{c}=16$ has only one stable dimension. There is a clear relation between the five characteristic times appearing in the evolution signal and the Hopf frequencies $\omega_{H q}$ of the two fixed points. The characteristic times associated with the node point, especially that of $\omega_{1}$, appear larger than the corresponding Hopf frequencies due to the presence of intermediate oscillations at the saddle frequencies. After a transient of a few tens of $\omega_{1}$ oscillations, during which the wave form structure presents very small changes, the signal looks periodic by repeating the complex sequence of oscillations within the period $2 \pi / \omega_{1}$. The Lyapunov exponents (in bits per unit time) have been calculated [18] and found equal to $-0.002,-0.015$, $-0.225,-4.16,-17.31,-18.13$.

Figure 7 presents a series of time evolutions for different $\mu_{c}$ values to show how the different oscillation modes appear on the wave form signal. The evolutions correspond to a six-dimensional system with the positive-defined sinusoidal function given by Eq. (A2) and characterized in Fig. 2(b). The steady-state diagram contains successive $S$-shaped branches and the reported evolutions are associated with a pair of fixed points of a high-order branch. The $p$ values of the fixed points are given in the caption, and their compari-
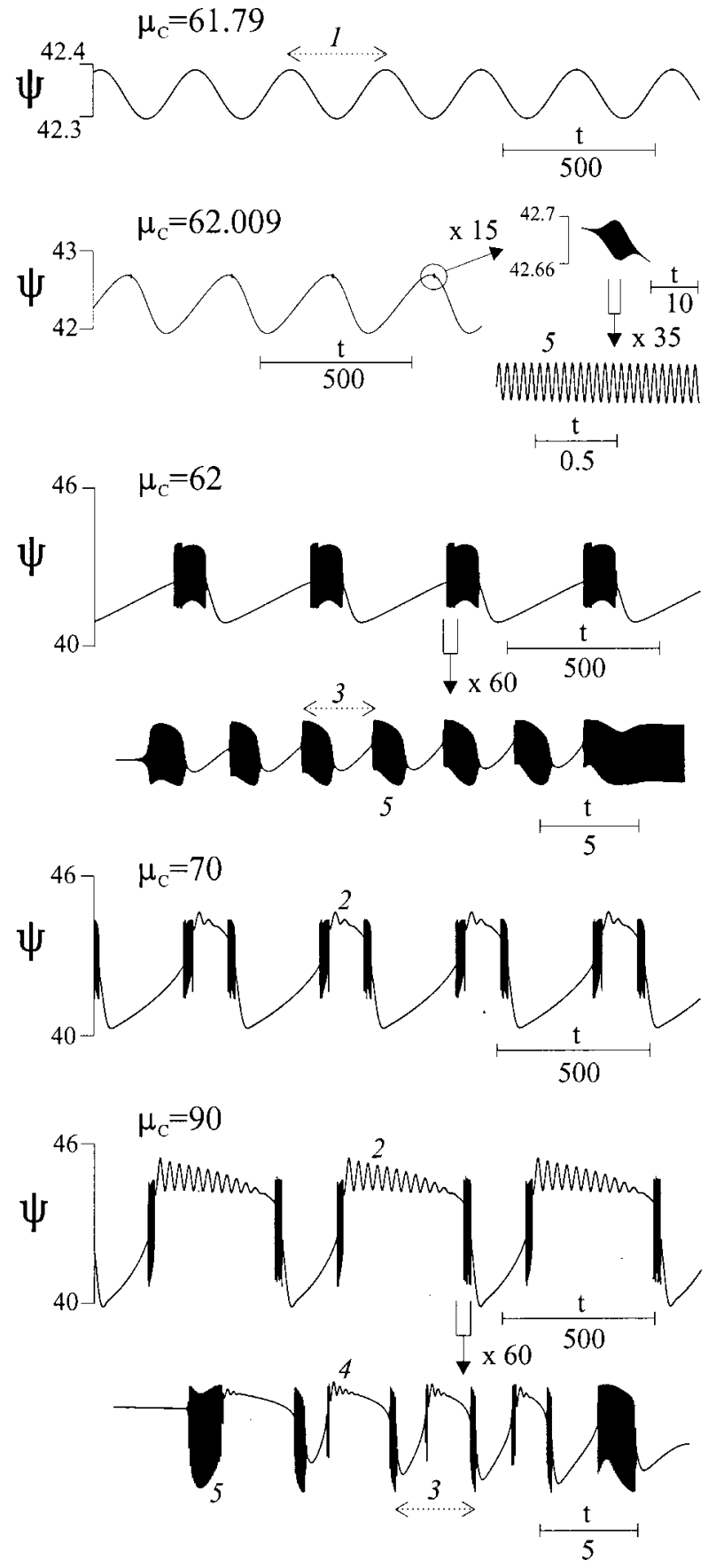

FIG. 7. Sequence of time evolutions for successive $\mu_{c}$ values showing how the wave form structures of the full instability behavior emerge. The nonlinear function is given by Eq. (A2) and the parameters are $\omega_{H q}=0.02,0.2,2,20,125, p_{H q}=-12,33,-18,40$, -17 , and $c_{1}=2 \omega_{H 5}$. From the top to the bottom, the $\bar{\psi}$ and $p$ values of the involved fixed points are (a) $\bar{\psi}=42.34, p=-12.002$; (b) and (c) $\bar{\psi}=42.35, p=-12.19 ;$ (d) $\bar{\psi}=45.4, p=16.3 ; \bar{\psi}$ $=42.7, p=-19.0$; (e) $\bar{\psi}=44.9, p=32.2 ; \bar{\psi}=43.1, p=-33.1$.

son with the $p_{H q}$ indicates the occurrence of the Hopf bifurcations.

The oscillations begin with a supercritical bifurcation of frequency $\omega_{H 1}$ occurring on the node point just below $\mu_{c}$ $=61.79$, but for $\mu_{c}=62.0085$ a minute component of fast oscillations appears from nothing in a certain place of the slow undulations (see the signal for $\mu_{c}=62.009$ ). The fast frequency is precisely equal to $\omega_{H 5}$, and the continuous fol- 
lowing of the stable orbit indicates that the appearance of such oscillations is not related to any local bifurcation. When the control parameter is increased, the localized structure of fast oscillations loses repetitivity and the orbit continuation breaks down at $\mu_{c}=62.0097$. After this point the system evolves toward a bigger attractor that in fact coexists with the previous orbit for a range of the control parameter beginning at $\mu_{c}=61.95$ (see the signal for $\mu_{c}=62$ ). The big attractor also contains a localized structure but with two characteristic frequencies: $\omega_{5}$ equal to $\omega_{H 5}$ and $\omega_{3}$ somewhat slower than $\omega_{H 3}$. The Hopf bifurcations at $\omega_{H 5}$ and $\omega_{H 3}$ occur on the node point for $\mu_{c}=67.56$ and 68.76 , respectively. The two bifurcations occur close enough so that the occurrence of a $\left(\omega_{3}, \omega_{5}\right)$ torus is likely. This suggest that the three-frequency wave form observed for $\mu_{c}=62$ may be tentatively interpreted as the $\omega_{1}$ stable orbit influenced by the flow associated with the invariant manifolds of a $\left(\omega_{3}, \omega_{5}\right)$ saddle torus. The possibility of an orbit on a stable three torus cannot be excluded, but there is no evidence for it.

The signal for $\mu_{c}=70$ shows the $\left(\omega_{3}, \omega_{5}\right)$ structure divided in two parts and denotes the approach of the attractor to the external saddle point by means of the oscillations of frequency $\omega_{2} \approx \omega_{H 2}$. For this signal we calculate the following Lyapunov exponents: $-0.0002,-0.055,-0.422$, $-8.37,-33.9,-201$. The influence of the saddle point is even more evident in the signal for $\mu_{c}=90$ where, in addition to the large number of $\omega_{2}$ oscillations, a new frequency $\omega \approx \omega_{H 4}$ appears mixed with the $\left(\omega_{3}, \omega_{5}\right)$ structure. The eigenvalues of the saddle point are $-0.0018 \pm i 0.199,0.0046$, $-1.44 \pm i 20.01$, and -719 , so that it is near to the $\omega_{H 2}$ bifurcation (at $\mu_{c}=91.35$ ) but relatively far from the $\omega_{H 4}$ bifurcation (at $\mu_{c}=103.84$ ).

The saddle eigenvalues reported above fulfill the conditions of the Shil'nikov theorem for homoclinic chaos [19] and the large number of $\omega_{2}$ oscillations indicates the proximity to homoclinicity. However, the evolution is periodic and we have verified that it remains periodic for different values of $\mu_{c}$ up to 96.07 , for which the signal contains $50 \omega_{2}$ oscillations of almost uniform amplitude denoting the presence of the saddle cycle created at the $\omega_{H 2}$ bifurcation. At $\mu_{c}=96.08$, the homoclinic connection has destroyed the attractor and the system has jumped to the oscillating state associated with a higher branch. With respect to the Shil'nikov theorem, it may be that either the complex dynamics will concentrate in an extremely narrow neighborhood of the homoclinic loop or that certain conditions concerning the saddle connection geometry are not fulfilled [19].

We have tried the continuous following of the saddle orbits created at the successive Hopf bifurcations of the fixed points. Notice from Fig. 2(b) that a given $p_{H q}$ value is found two times on the same steady state branch and the continuation of each orbit has been initiated from the Hopf bifurcations at the two branch sides. The bifurcations have always been found to be supercritical. The orbit that emerged from the $\omega_{H 5}$ bifurcation shows a nearly sinusoidal evolution but the frequency decreases, stabilizing near half of $\omega_{H 5}$. This is a remarkable feature because the faster oscillations appearing on other obits have a frequency precisely equal to $\omega_{H 5}$. One of the orbit multipliers grows very strongly and the continuation becomes rather slow so that it has been followed in limited ranges near the Hopf points only. The orbit $\omega_{H 3}$ has
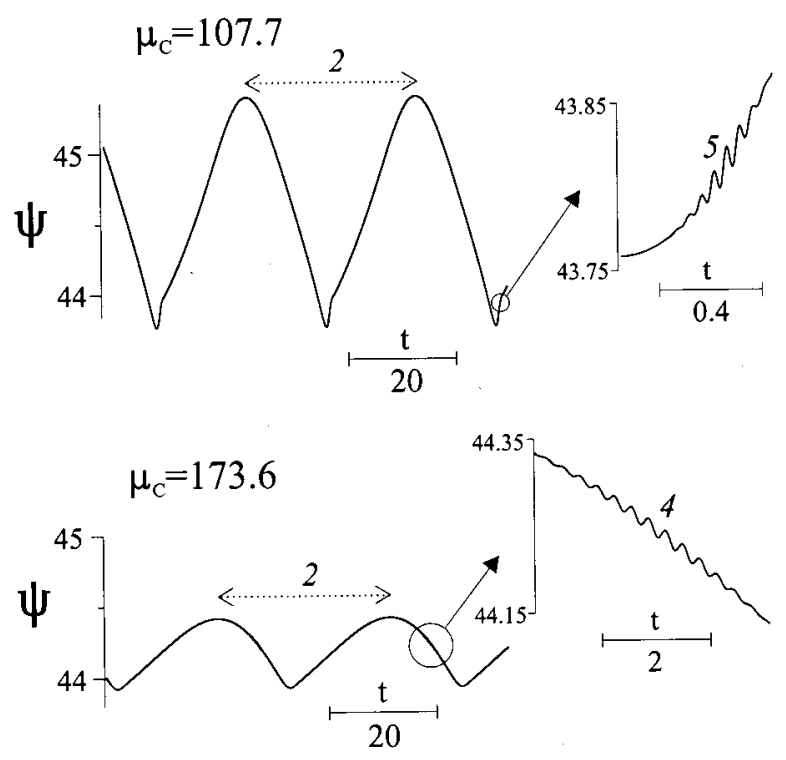

FIG. 8. Time evolution of the saddle orbits created at the $\omega_{H 2}$ bifurcations occurring on the saddle point for $\mu_{c}=91.35$ and 186.78, respectively.

not been detected. The orbit $\omega_{H 4}$ has been continuously followed up to its disappearance in the reverse Hopf bifurcation at $\mu_{c}=177.98$, always showing a nearly sinusoidal evolution of frequency almost equal to $\omega_{H 4}$. The continuation of the orbit $\omega_{H 2}$ is more delicate because the evolution incorporates high-frequency oscillations at $\omega_{H 5}$ and $\omega_{H 4}$, as illustrated in Fig. 8. The orbit multipliers do not denote the occurrence of local bifurcations. These results show how the mode mixing also affects the saddle orbits.

The full instability behavior based on rather different oscillation frequencies usually exhibits robust wave form structures that repeat identically if a long enough transient is discarded. Nevertheless, chaotic evolutions may also be found by increasing the control parameter enough. For instance, Fig. 9 presents an example of chaotic evolution obtained from the same system as Fig. 7 for a higher value of $\mu_{c}$. For this case we calculate the following spectrum of Lyapunov exponents: $0.0268,-0.00184,-0.0498$, $-2.269,-6.031,-1072$. The aperiodic evolution arises from the irregular repetition of the complex wave form structure, and it is worth remarking that the wave form complexity cannot be attributed to chaos. In other words, the fine tuning of $\mu_{c}$ leads to periodic states in which the wave form complexity remains.

Figure 10 illustrates self-similarity features of the full instability evolutions at two different levels, one with respect to the time scale and another with respect to the system dimension. Self-similarity is also apparent in the wave forms of Figs. 1 and 7. Notice that the oscillations associated with either the node or the saddle points maintain their roles along the similarity scale. The reported results contain a reduced number of similarity levels but there is no reason for a limit in the system dimension, and it will therefore be interesting to investigate the self-similarity of the full instability behavior with respect to both the time and frequency domains for high $N$ values.

The various signals of Fig. 10 correspond to the same 


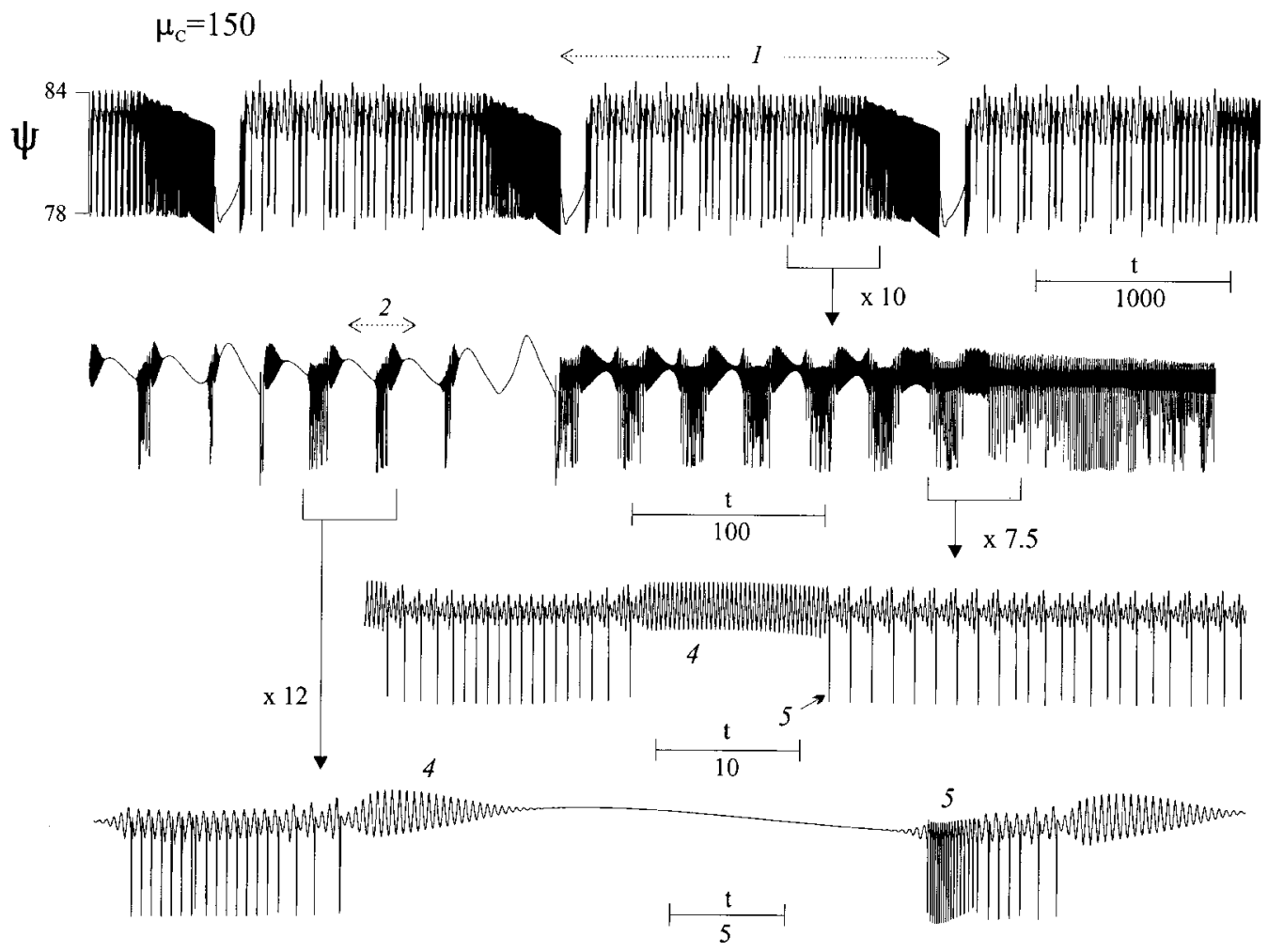

FIG. 9. Chaotic evolution obtained for the same case as in Fig. 7 but for a higher value of the control parameter.

nonlinear function and same $\mu_{c}$ value so that the systems of different dimension have the same set of fixed points with identical $\bar{\psi}$ and $p$ values. The evolutions are always associated with the same pair of saddle-node fixed points. Notice the unique $\psi$ scale and the three zoom levels of the time scale employed in the representations. The first zoom level of case (c) corresponds to the second level of the others cases. The parameters of the various systems have been determined by imposing a common set of the $\omega_{H}$ and $p_{H}$ values and a similar degree of vector field divergence. For instance, by taking out either the two faster or the two slower frequencies of system (a) with $N=8$, we have either system (b) or system (c), both with $N=6$. Similarly, the system (d) with $N=5$ has the same Hopf bifurcations as system (b) except for that at the higher frequency. In this way we obtain systems of different dimension in which the linear stability behavior of a saddle-node pair of fixed points is partially identical. Another significant property is the vector field divergence, and we have chosen relatively similar values of $c_{1}$ with respect to the higher frequency for the different systems of Fig. 10.

Figure 11 corresponds to a six-dimensional system with a nonlinear function sustaining a symmetric pitchfork bifurcation and has the same values for $\omega_{H q}, p_{H q}$, and $c_{1}$ as in Fig. 1. The nonlinear function is given by Eq. (A3) and characterized in Fig. 2(c). The pitchfork produces two stable nodes and a saddle with one unstable dimension and, for the range of $\mu_{c}$ values considered in Fig. 11, the system presents the trio of fixed points only. These points experience Hopf bifurcations at $\mu_{c}=2\left(\omega_{H 1}\right), 2.35\left(\omega_{H 3}\right), 3\left(\omega_{H 2}\right)$, and 3.2 $\left(\omega_{H 4}\right.$ and $\left.\omega_{H 5}\right)$, approximately. The first bifurcation, at $\omega_{H 1}$, occurs on both nodes and creates a pair of coexisting attractors located symmetrically with respect to the phase space origin. The time evolution for $\mu_{c}=2.25$ describes one of these attractors and it already contains oscillations at $\omega_{2}, \omega_{3}$, and $\omega_{4}$. The signal for $\mu_{c}=2.5$ describes a two-lobed attractor resulting from the gluing together of the two previous attractors through two simultaneous homoclinic connections of the saddle point. The node points have already made the second Hopf bifurcation at $\omega_{H 3}$, and now the signal includes oscillations at the five frequencies. For $\mu_{c}=3.5$ the node points have experienced the third bifurcation at $\omega_{5}$, while the intermediate saddle has made the bifurcations at $\omega_{2}$ and $\omega_{4}$. The time evolution now shows a rich wave form structure pointing out the full instability behavior of the system around a node-saddle-node trio of fixed points. Notice that the evolution is also based on five oscillation frequencies, i.e., $N$ -1 frequencies, because the two node points suffer the Hopf bifurcations with the same $\omega_{H}$ and $p_{H}$ values.

Figure 12 illustrates the influence of the vector field divergence on the full instability behavior and, in essence, it shows how the dissipation discriminates the oscillation frequencies associated with either the node or the saddle points and how it regulates their participation in the observed time dynamics. Notice the absence of $\omega_{2}$ and $\omega_{4}$ for $c_{1}=0$ and $c_{1}=60$, and the small vestige of $\omega_{5}$ for $c_{1}=3.6 \omega_{H 5}$. The represented evolutions correspond to different sixdimensional systems that have in common the same nonlinear function (the same as in Fig. 11), the same $\mu_{c}$ value, and the same set of $p_{H q}$ and $\omega_{H q}$ for the five Hopf bifurcations, but different values of the coefficient $c_{1}$. Since one of the oscillation frequencies is clearly higher than the others, the vector field divergence may be characterized by Eq. (B3) and directly associated with $c_{1}$.

Thus the evolution signals of Fig. 12 corresponds to systems of successively increasing dissipation, from the conser- 

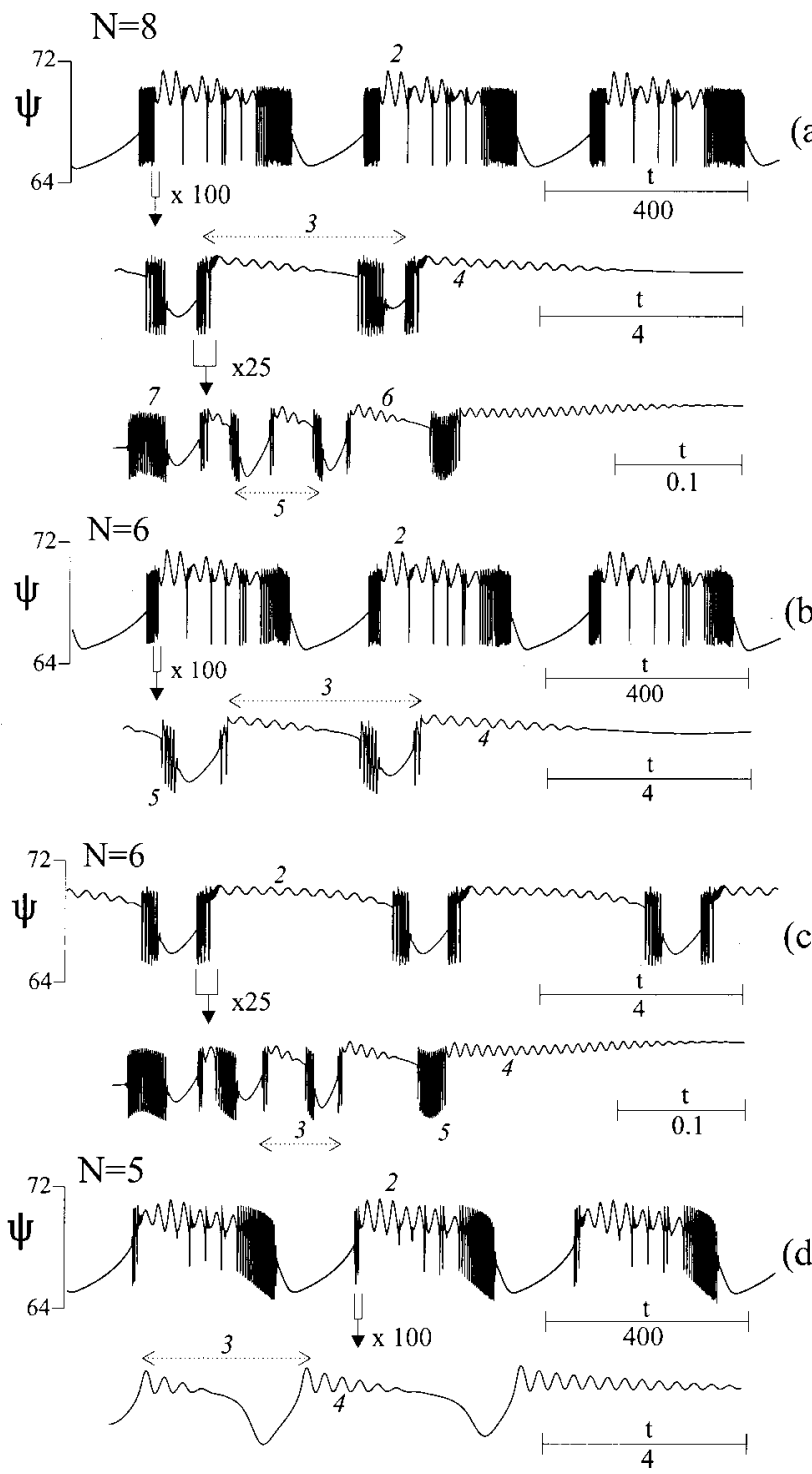

FIG. 10. Time evolutions from different systems illustrating self-similarity with respect to both the time scale and the system dimension. The nonlinear function is the same as in Fig. 7, $\mu_{c}$ $=120$, and other parameters are (a) $N=8, \omega_{H q}=0.0209,0.251$, $2.86,18.9,126,700,5000, p_{H q}=-12,33,-18,45,-18,45$, $-18, c_{1}=2.15 \omega_{H 7}$; (b) $N=6, \omega_{H q}=0.0209,0.251,2.86,18.9$, $126, p_{H q}=-12,33,-18,45,-18, c_{1}=1.98 \omega_{H 5}$; (c) $N=6, \omega_{H q}$ $=2.86,18.9,126,700,5000, p_{H q}=-18,45,-18,45,-18, c_{1}$ $=2.15 \omega_{H 5}$; (d) $N=5, \omega_{H q}=0.0209,0.251,2.86,18.9, p_{H q}=-12$, $33,-18,45, c_{1}=0.69 \omega_{H 4}$.

vative case $c_{1}=0$ to the strongly dissipative of $c_{1}=3.6 \omega_{H 5}$. All of the systems have the same steady-state branching diagram as a function of $\mu_{c}$ and the same distribution of $p$ values over that diagram, and the fixed points with $p=p_{H q}$ are nonhyperbolic with eigenvalues $\pm i \omega_{H q}$. In the $k_{j}$ space, the straight line describing the fixed points moves with $c_{1}$ but, for $c_{1} \neq 0$, maintains its qualitative relation with the surfaces $\{0\}$ and $\{ \pm i \omega\}$; i.e., the line crosses the same instability regions and produces qualitatively equivalent branching diagrams. This means that tall the dissipative systems experience the Hopf bifurcations as in the case of Fig. 11. In particular, the represented evolutions correspond to $\mu_{c}=3$ for which the pair of node points have already done the bi-

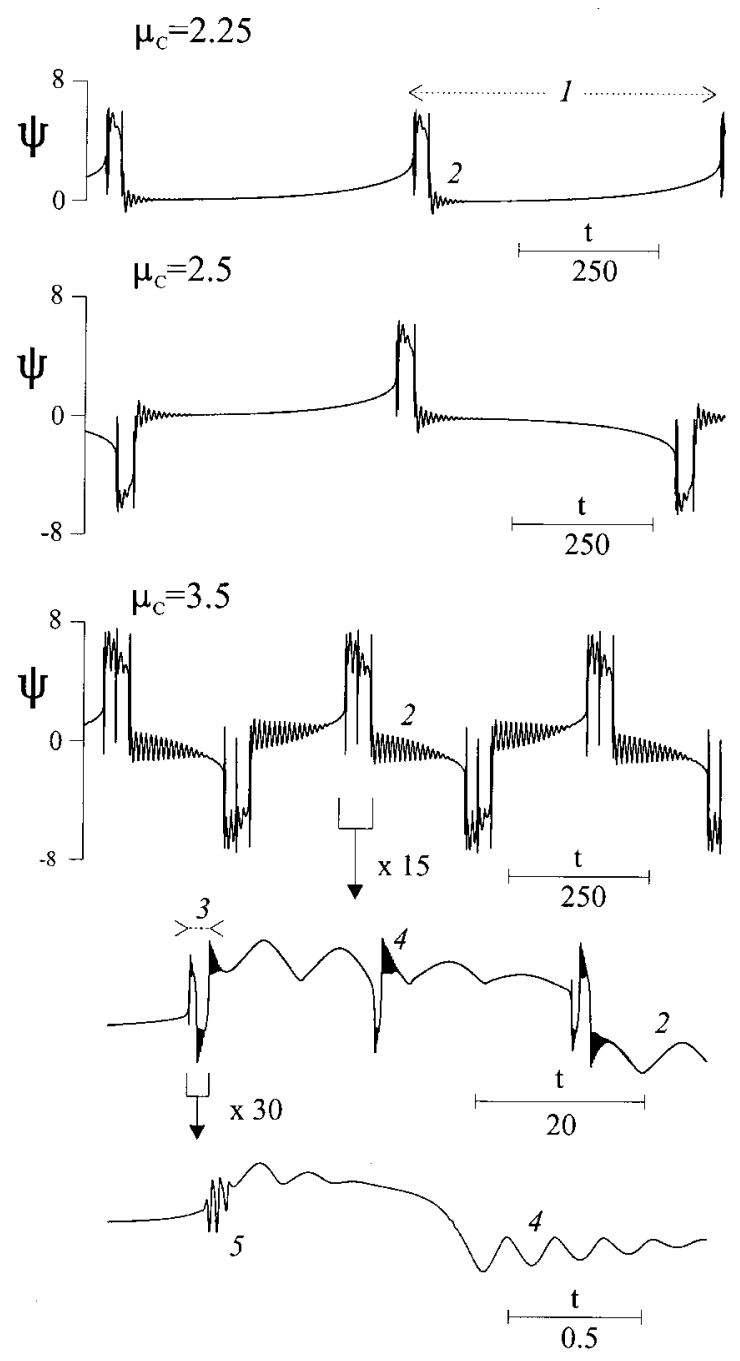

FIG. 11. Time evolutions for successive $\mu_{c}$ values illustrating the full instability behavior around a node-saddle-node trio of fixed points generated in a symmetric pitchfork bifurcation. The nonlinear function is $g(\psi)=\sin \psi$, and other parameters are the same as in Fig. 1

furcations at $\omega_{H 1}$ and $\omega_{H 3}$ and the saddle has done the bifurcation at $\omega_{H 2}$.

The conservative case behaves differently. In the limit $c_{1}=0$, the straight line does not cross the surface $\{ \pm i \omega\}$ but penetrates within it and within regions of high-order degeneracies. This means that the fixed points remain highly nonhyperbolic for long ranges of the $\mu_{c}$ parameter and the Hopf bifurcations do not occur. Concretely, the "node points" are three-dimensional toroidal centers and the "saddle point" is a two-dimensional toroidal center with additional onedimensional stable and unstable manifolds of equal eigenvalues. There are no attractors and every set of initial conditions leads to a particular trajectory. In the example of Fig. 12, the trajectory evolves around one of the lateral points, but trajectories around the three fixed points are also possible. In any case, the evolution contains only three oscillation frequencies and they are always of the order of $\omega_{H 1}, \omega_{H 3}$, and $\omega_{H 5}$.

The numerical results presented up until now correspond to systems exhibiting the full instability behavior on the basis of really different frequencies, i.e., about an order of magni- 

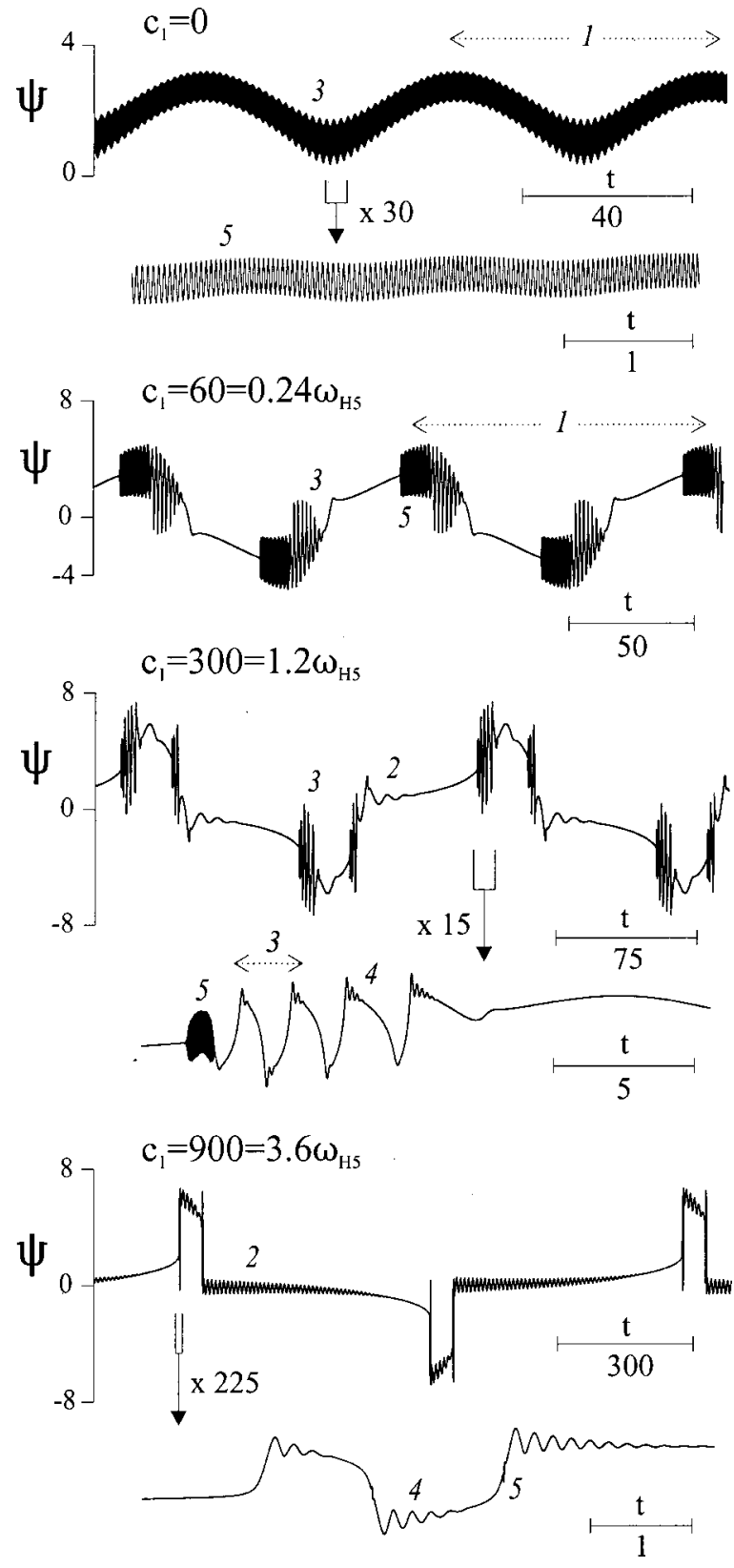

FIG. 12. Influence of the level of dissipation on the full instability evolutions. The signals correspond to the same system as in Fig. 11, except for the coefficient $c_{1}$ that is varied from 0 to $3.6 \omega_{H 5}$, and $\mu_{c}=3$. The trajectory for $c_{1}=0$ corresponds to the initial conditions $x_{N}=10^{-4}$ and $x_{j \neq N}=0$. Notice the different time scales for the various signals.

tude between consecutive frequencies, and Fig. 13 illustrates how the dynamics become more irregular when the oscillation frequencies become closer. The evolution of Fig. 13(a) must be compared with that of Fig. 7 for $\mu_{c}=90$, because the unique differences between both situations are the Hopf frequencies $\omega_{H q}$, now sequenced with a factor of three, and the coefficient $c_{1}$, which maintains the same relation $c_{1} / \omega_{H 5}$ in order to achieve a similar level of dissipation. Similarly, the signal of Fig. 13(b) has to be compared with that of Fig. 12 with $c_{1} / \omega_{H 5}=1.2$. The signals of Fig. 13 describe the evolution after a time length 50 times longer than the represented interval in order to avoid transitory effects. The Lyapunov spectrum calculated for case (a) is 0.000848 ,
$0.000414,-0.0159,-0.0989,-0.143,-8.20$, and for case (b) is $0.0271,-0.0005,-0.0330,-0.326,-0.729$, -21.88 . Thus signal (b) is clearly chaotic while (a) may be associated with a quasiperiodic orbit.

Finally, it is worth mentioning that the thresholds $p_{H q}$ can be employed to regulate the relative position of the Hopf bifurcations and the proximity of the system to particular eigenvalue degeneracies. The analysis of their influence on the observed dynamics would provide a large perspective about the full instability behavior.

\section{DISCUSSION}

The linear stability analysis can indicate the occurrence of successive Hopf bifurcations on the fixed points when the parameter is varied and the nonlinearity of the system will usually guarantee the generation of successive limit cycles. One of the cycles will probably be initially stable while the rest will be saddle cycles of different types. Of course, a variety of secondary processes of both local and global nature can occur to any of these periodic orbits and a large variety of observable behaviors may then be expected. Nevertheless, the numerical simulations for systems in the form (2), (4), and (5), with different nonlinear functions and for different dimensions, confirm the essential role of the steadystate bifurcations in determining the time dynamics observed in the full instability regimes. Although the sequence of processes yielding the underlying attractor may be rather complex and dependent on the circumstances, it seems that some generic mechanisms are responsible for the mode mixing observed in the time dynamics, at least in the case of a nonlinear function of a single variable in the form $(5 b)$.

\section{A. Secondary bifurcations and mixing mechanisms}

The basic mechanism of nonlinear mixing is simply the attractor intertwinement around the unstable manifold of a neighboring saddle limit set. In this way, the time dynamics of the attractor incorporates oscillations at the saddle frequencies without requiring any bifurcation [20]. This happens, for instance, in the Shil'nikov-type attractors formed when a stable limit cycle approaches an external saddle focus or limit cycle. More generally, we conjecture that this mechanism can work with the variety of saddle sets emerged from the saddle-node pair of fixed points, because all of them have a branch of the unstable manifold ending toward the attractor. The approach to well defined places of the attractor would explain the characteristic wave forms formed by a succession of packets of different frequencies. The intertwinement can affect the saddle orbits also and, in fact, the nonlinear mode mixing of the full instability behavior may be considered like a global process affecting the flow of an extended region of the phase space where the invariant sets will form accordingly. It must be stressed, however, that we have not determined the unstable manifolds in the numerical simulations for $N>3$, and this will be necessary for verifying the proposed mixing mechanism.

A deeper combination of oscillation modes can occur through the Naimark-Sacker bifurcation, usually called the torus or secondary Hopf bifurcation. In this case, the invariant torus sustains true multifrequency oscillations and complex dynamics associated with resonances and torus break- 

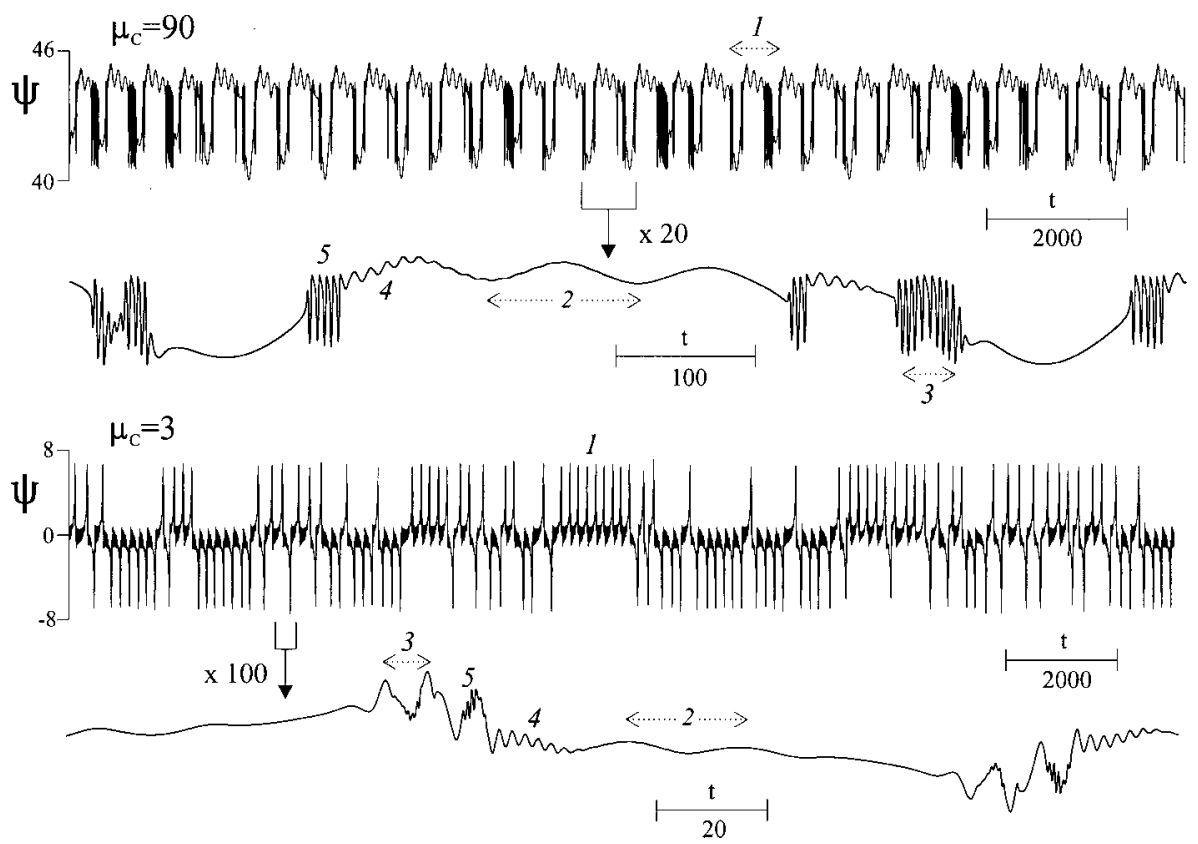

down problems are intrinsically possible. Each fixed point of the saddle-node pair may yield tori with multimode oscillations related to the Hopf frequencies of that point and, in principle, the number of such bifurcations determines the dimension of the highest-order torus. In the parameter space, the $q$-torus bifurcation emerges from the degeneracy $\left\{ \pm \omega_{1}, \ldots, \pm i \omega_{q}\right\}$ of the fixed point eigenvalues. In particular, the two-torus bifurcation emerges from $\left\{ \pm i \omega_{1}, \pm i \omega_{2}\right\}$ and the corresponding universal unfoldings have been investigated in detail $[1,21]$. It has been shown that, in certain cases, this four-dimensional degeneracy can produce an additional three-torus bifurcation with the third frequency not directly related to the fixed point bifurcations. Similarly, the three-dimensional degeneracy $\{0, \pm i \omega\}$ can produce a twotorus bifurcation where the second frequency seems again independent of the fixed points [1]. These tori cannot be associated with a specific fixed point and a generalization to higher-order eigenvalue degeneracies suggests the possibility of tori with dimensions up to $N-1$, i.e., the highest order in $N$ dimensions. Nevertheless, when numerically observed $[22,23]$, these tori appear very fragile and in extremely narrow regions of the parameter space.

In conclusion, we find reason to suspect that the full instability behavior will happen in parameter space regions with a relative abundance of torus bifurcations yielding multifrequency oscillations based on the Hopf bifurcations of one of the fixed points. The node-point family of cycles and tori contains at least one attractor [24], while the rest of this family and the saddle-point family are saddles. With the variation of a control parameter, the attractor grows and transforms while the neighboring saddle sets move their stable and unstable manifolds in a process that underlies a variety of possible homoclinic and heteroclinic connections. The time dynamics of the attractor incorporates the oscillatory motions of the various saddles in a degree that is very sensitive to how near the corresponding homoclinicities are. Of course, complex and dense sequences of bifurcations yielding to homoclinic chaos can also occur during the mixing process [19]. However, the period-doubling and cyclic saddle-node bifurcations are not able to introduce independent characteristic frequencies and are therefore not essential for explaining the full instability behavior, at least in a first approximation. What is more relevant is the possibility of attractor destruction after a homoclinic connection.

\section{B. Basic set of fixed points}

We want to remark here why we consider a saddle-node pair of fixed points. Of course, there are nonlinear systems possessing only one fixed point and the full instability in this case corresponds to $N / 2$ Hopf bifurcations on this point, if $N$ is even, or to $(N-1) / 2$ if $N$ is odd. However, the coexistence of steady states is one of the most significant features of nonlinear dynamics and it is then convenient to consider this possibility for a more general overview. What basic set of fixed points has to be considered depends essentially on the directional structure of the nonlinear part of the vector field, i.e., on the number of linearly independent components of uniform direction in which the nonlinear vector field can be decomposed.

For one-directional nonlinear vector fields, the fixed points appear in phase space aligned on a straight line and in an alternate sequence of saddle-node type. The saddle separatrices determine the attraction basins of the nodes and the basic dynamical phenomena will be associated with an attractor arising form one of the nodes and growing in the presence of the nearest saddle. Of course there are also mechanisms for producing attractors associated with a larger number of fixed points. On the one hand, it is possible that the attractor will grow under the influence of two neighboring saddle points located at the opposite sides. On the other hand, attractors developed separately at the two sides of a saddle separatrix can become glued together through successive homoclinic bifurcations destroying the previous attractors and creating the hybrid ones. This kind of gluing is well known from the systems with a symmetric pitchfork bifurcation, in which the homoclinic connections happen simulta- 
neously at the two saddle sides [25], but it can also occur in more general situations [26]. For systems whose fixed points appear on a straight line in the $k_{j}$ space, the various node $\left(k_{N}>0\right)$ and saddle $\left(k_{N}<0\right)$ points will generate the same oscillation frequencies and the time evolutions will always be based on the $N-1$ oscillation modes, independent of the number of underlying fixed points. In a more general case, the oscillation frequencies generated from different nodes or saddles can be different and the time evolutions will incorporate additional modes by an increase in the number of fixed points.

The situation becomes more complex for vector fields with a multidirectional nonlinear part like that defined by the superposition of $n$ nonlinear vector fields based on linearly independent scalar functions and directions. In this case, the fixed points may extend in phase space distributed within a subspace of dimension $n$, related ones with others through $n$ differently oriented $\lambda=0$ bifurcations. The basic set of fixed points may include up to $2^{n}$ points, one of which is a stable node and the rest are saddles defining one of the corners of its attraction basin. This set of points can sustain a large number of Hopf instabilities but it is not evident to what extent the corresponding oscillatory motions can manifest together in the time dynamics.

\section{CONCLUDING REMARKS}

We have verified that the combination of oscillatory motions in a nonlinear dynamical system can yield complex time evolutions in a way that evokes the Landau proposal for tentatively explaining the origin of turbulence [3]. This behavior has been found in $N$-dimensional systems able to exploit the instability capabilities of their fixed points to a large extent. The oscillations emerge in association with Hopftype bifurcations and the complexity arises from (i) the number of different oscillation modes, and (ii) the variety of forms through which the nonlinear mechanisms combine the oscillation modes. The second point marks the essential difference with the Landau scenario; i.e., the mode mixing mechanisms of nonlinear dynamics are richer than the superposition of oscillatory instabilities by successive torus bifurcations and they can introduce irregularity in the time evolution by affecting both the relative phases and amplitudes in a complex manner. In addition, a set of coexisting fixed points can generate more than the $N / 2$ characteristic frequencies of a sequence of torus bifurcations. In other words, the nonlinear dynamical systems can have enough mechanisms for developing the Landau intuition about the emergence of irregularity through oscillatory superpositions. Another question is whether this irregular evolution may be related to the origin of turbulence in fluids.

On the other hand, the irregular succession of undulations of different characteristic times forming the full instability wave forms can repeat regularly and even periodically. This fact indicates that the high degree of instability behavior represents a way toward creating irregular and complex evolutions independent of chaos. The complexity degree of the full instability signals can be emphasized by considering, for instance, the case of Fig. 10(a) and realizing that if the characteristic time of the fast oscillations would be one day, the longest time defining the period would be about one thousand years, after which time the system would repeat the same irregular sequence of daily undulations again. It will be interesting to investigate the properties of the irregular evolutions of the fully unstable systems for higher dimensions and to compare them with the well-known properties of the chaotic dynamics.

In this paper, the full instability behavior has ben investigated in particular classes of dynamical systems and it is important to ask how general the phenomenon is. According to our interpretation, we find reason to suspect that the occurrence of the various Hopf bifurcations in a restricted parameter domain will always be associated with the development of nonlinear mechanisms of mode mixing. Nevertheless, the complexity of the process and the characteristic features of the time dynamics will probably change with the structure of the nonlinearities. For instance, the fact that the oscillation modes appear with the same frequencies as the Hopf bifurcations of the fixed points may perhaps be peculiar for nonlinear functions in the form (5).

Another significant question is to what extent the full instability behavior may be relevant for the study of real systems to which the concept of dynamical system is trying to be applied. The presence of oscillatory behaviors of dynamical nature is indeed evident in biology, economics, and sociology. In certain cases, for instance, in living systems [27], a variety of oscillatory processes with different time scales occur at different levels or in different parts of a given system. Some of these processes develop autonomously, but interrelations and oscillatory mixing can also occur [28]. The description of such a mixing at the level of a dynamical system would probably involve the nonlinear combination of oscillations associated with a set of neighboring fixed points, i.e., a system exploiting the instability capabilities of its fixed points to a certain extent.

By following the Landau proposal we must also consider the turbulence phenomena. With respect to this, the selfsimilarity properties observed in the full instability signals may be particularly suggerent. Such properties are intrinsically related to the organization of the mode mixing processes around the structure of fixed points and constitute a robust feature. On the other hand, the dynamical systems typically obtained by reduction of the partial differential equations associated with turbulent problems [29] always present (i) a multidirectional nonlinear part of the vector field with the number of independent nonlinear functions as large as the order of the system, and (ii) the participation of a large number of dynamic variables within every nonlinear function. This means the occurrence of complex structures of fixed points and possibilities for exploiting the instability capabilities of these points, two different and complementary ways through which complex behavior may emerge in dynamical systems and whose interrelation we find interesting to investigate.

Finally, as a matter of fact, we want to recall that the full instability behavior has been observed in the BOITAL devices [8] and that these devices are really simple objects; in essence, they are $\mathrm{N}$-layer sandwiches of glasses and liquids placed between two mirrors and irradiated by a light beam. 


\section{ACKNOWLEDGMENT}

This work has been supported by the PB98-899 of the Spanish CICYT.

\section{APPENDIX A: NONLINEAR FUNCTIONS USED IN THE NUMERICAL SIMULATIONS}

We have used the following nonlinear functions of a single variable:

$$
g(\psi)=\mu_{1}+\exp \left[-\frac{\left(\psi-\mu_{2}\right)^{2}}{\mu_{3}}\right]
$$

with $\mu_{1}=0.1$ and $\mu_{2}=\mu_{3}=10$, to obtain the results of Fig. 1 ;

$$
g(\psi)=\frac{\mu_{1}+\mu_{2} \cos \psi}{\mu_{3}+\cos \psi}
$$

with $\mu_{1}=-1.25, \mu_{2}=1.06$, and $\mu_{3}=-1.86$, to obtain the results of Figs. 7-10 and 13(a); and

$$
g(\psi)=\sin \psi,
$$

to obtain the results of Figs. 11, 12, and 13(b). These nonlinear functions are represented in Figs. 2(a), 2(b), and 2(c), respectively, together with the corresponding steady-state branching diagrams and distribution of $p$ values. The diagrams describing both $\bar{\psi}$ and $p$ as a function of $\left(d_{N} / c_{N}\right) \mu_{c}$ are independent of $N$ and the coefficients $c_{j}$ and $d_{j}$. If $c_{N}$ $=d_{N}$ the diagrams will then be determined exclusively by the nonlinear function.

The Gaussian function, Eq. (A1), produces a $S$-shaped branching diagram with a single hysteresis cycle and with a limited range of $p$ values. The periodic function of Eq. (A2) is a positive-defined sinusoidal function describing the light interferences in the low-finesse, high-contrast cavities of the BOITAL devices [9]. The periodicity of the nonlinear function produces successive $S$-shaped steady-state branches with successively broader distribution of $p$ values. This means that the required values of $p$ are always available by going to higher branches. The sinusoidal function of Eq. (A3) has the peculiarity that fulfills the conditions for a pitchfork bifurcation and its periodicity leads to additional saddle-node branches in the steady-state diagram. With this function the system is invariant under the sign inversion of the full set of variables.

\section{APPENDIX B: SYSTEMS IN THE FORM (2), (4), AND (5) FOR THE FULL INSTABILITY BEHAVIOR}

By introducing Eq. (11) in Eqs. (14) we can obtain the $p_{H}$ and $\omega_{H}$ values of the straight line intersections with $\{ \pm i \omega\}$ for a given set of coefficients $c_{j}$ and $d_{j}$, but the achievement of full instability situations is rather difficult in this way. It is also possible to work in the opposite way by previously (and properly) selecting the $p_{H}$ and $\omega_{H}$ values of the $N-1$ Hopf bifurcations and determining the corresponding set of param- eters $c_{j}$ and $d_{j}$. This gives two decoupled sets of $N-1$ equations, involving coefficients with even and odd labels, respectively. The total number of unknowns is $2 N$ and, therefore, two additional conditions are required. We usually take $d_{N}=c_{N}$ and choose a value for $c_{1}$. The former condition is not a restriction because it can always be done with the proper rescaling [17] and has the advantage of making the steady-state branching diagram depending on the $g(\psi)$ only. The later condition responds to the fact that, having considered $d_{N}=c_{N}$ the system of equations with odd label coefficients has $N$ unknowns and one of them must be predetermined. We take $c_{1}$ but any other odd label coefficients will be equivalent because the system of equations is scale invariant with respect to the unknowns. Thus the selected values for $\omega_{H}$ and $p_{H}$ determine the odd label coefficients with a free common scale factor that is defined by the preselected value of one of them. This scale factor is relevant because it affects the vector field divergence. In effect, the divergence of the canonical system (2) with a nonlinear function in the forms (4) and (5) is given by

$$
\operatorname{div} F=-c_{1}+p(\psi) \frac{c_{N}}{d_{N}} d_{1}=-k_{1}(\psi),
$$

where $p$ and $k_{1}$ are defined as in Eqs. (9) and (11), respectively, but for any point of the phase space. In particular, for systems having one of the frequencies $\omega_{H}$ clearly higher than the rest, it may be seen that

$$
c_{1} \approx p_{H F} \frac{c_{N}}{d_{N}} d_{1}
$$

were $p_{H F}$ corresponds to the fast frequency $\omega_{H F}$, and the effective dissipation along a given trajectory may be then characterized by

$$
\frac{\langle\operatorname{div} F\rangle}{\omega_{H F}} \approx-\frac{c_{1}}{\omega_{H F}}\left(1-\frac{\langle p\rangle}{p_{H F}}\right),
$$

where the brackets denote the average over the trajectory. Thus for a given nonlinear function $g(\psi)$, the election of $c_{1}$ allows us to adjust the dissipation degree of the $\mu_{c}$ family of systems that in the $k_{j}$ space intersects the surface $\{ \pm i \omega\}$ with the preselected set of values $\omega_{H}$ and $p_{H}$.

The selection of the $\omega_{H}$ and $p_{H}$ values must be done properly. A useful guideline for obtaining systems with the full instability behavior is to choose the ordered sequence of frequencies $\omega_{H}$ alternatively associated with either $p_{H}<0$ or $p_{H}>1$, i.e., with either the node or the saddle point. The condition $p_{H}<0$ instead of $p_{H}<1$ facilitates that the point of coordinates $\left(c_{j}\right)$ having $p=0$ will appear in the region with zero unstable dimensions and that the zero eigenvalue bifurcations will involve fixed points with zero and one unstable dimensions. On the other hand, the selection of the $p_{H}$ values allows us to achieve particular situations. For instance, a straight line crossing the degeneracy $\left\{ \pm i \omega_{1}, \ldots, \pm i \omega_{q}\right\}$ may be obtained by imposing $q$ Hopf bifurcations at the same $p$ value. 
[1] J. Guckenheimer and P. Holmes, Nonlinear Oscillations, Dynamical Systems, and Bifurcations of Vector Fields (Springer, New York, 1983).

[2] S. Wiggins, Introduction to Applied Nonlinear Dynamical Systems and Chaos (Springer, New York, 1990).

[3] L. D. Landau, C. R. (Dokl.) Acad. Sci. USSR 44, 311 (1944).

[4] D. Ruelle and F. Takens, Commun. Math. Phys. 20, 167 (1971); S. Newhouse, D. Ruelle, and F. Takens, ibid. 64, 35 (1978).

[5] Half of the bifurcations occur on each point if $N$ is odd, while one more bifurcation happens on the initially stable point if $N$ is even.

[6] In fact, only one attractor can emerge from the variety of Hopf bifurcations of a saddle-node pair of fixed points, but additional attractors may arise from secondary processes.

[7] R. Herrero, R. Pons, J. Farjas, F. Pi, and G. Orriols, Phys. Rev. E 53, 5627 (1996).

[8] J. Rius, M. Figueras, R. Herrero, F. Pi, and G. Orriols (unpublished).

[9] J. I. Rosell, J. Farjas, R. Herrero, F. Pi, and G. Orriols, Physica D 85, 509 (1995).

[10] J. Farjas, J. I. Rosell, R. Herrero, R. Pons, F. Pi, and G. Orriols, Physica D 95, 107 (1996).

[11] G. Duffing, Erzwungene Schwingungen bei Veränderlicher Eigenfrequenz, (Vieweg, Braunschweig, 1918).

[12] B. van der Pol and J. van der Merk, Nature (London) 120, 363 (1927).

[13] V. Volterra, Leçons Sur la Téorie Mathématique de la Lutte Pour la Vie (Gauthiers-Villars, Paris, 1936).

[14] O. E. Rössler, Phys. Lett. 57A, 397 (1976).

[15] L. O. Chua, C. W. Wu, A. S. Huang, and G.-Q. Zhong, IEEE Trans. Circuits Syst. I: Fundam. Theory Appl. 40, 732 (1993).

[16] A nonsingular transformation between systems (1) and (2) exists if and only if $\operatorname{rank}\left[b, A b, A^{2} b, \ldots, A^{(N-1)} b\right]=N$. See, e.g., S. Barnet and R. G. Cameron, Introduction to Mathematical Control Theory, 2nd ed. (Clarendon, Oxford, 1985), p. 85.

[17] The transformation $\quad x_{j} \rightarrow\left(d_{N} / c_{N}\right) x_{j}, d_{j} \rightarrow\left(c_{N} / d_{N}\right) d_{J}, c_{j}$ $\rightarrow c_{j}, \mu_{c} \rightarrow\left(d_{N} / c_{N}\right) \mu_{c}$ leaves system (2) with Eqs. (4) and (5) invariant but it makes $d_{N}=c_{N}$.

[18] A. Wolf, B. Swift, H. L. Swinney, and J. A. Vastano, Physica D 16, 285 (1985).

[19] L. P. Shil'nikov, Int. J. Bifurcation Chaos Appl. Sci. Eng. 4, 489 (1994).

[20] Complex sequences of bifurcations may occur near homoclinicity when the saddle set fulfills proper conditions [19], but mode mixing happens independently.

[21] For a review see V. I. Arnold, V. S. Afrajmovich, Yyu. S. Il'yashenko, and L. P. Shil'nikov, in Dynamical Systems V, edited by V. I. Arnold (Springer-Verlag, Berlin, 1994), pp. $1-205$.

[22] P. Gaspard, Physica D 62, 94 (1993).

[23] X.-J. Wang and G. Nicolis, Physica D 26, 140 (1987).

[24] Provided that a stable fixed point exists at a certain moment.

[25] P. Glendinning, in New Directions in Dynamical Systems, edited by T. Belford and J. Switf (Cambridge University Press, Cambridge, 1988), p. 120.

[26] R. Herrero, J. Farjas, R. Pons, F. Pi, and G. Orriols, Phys. Rev. E 57, 5366 (1998).

[27] A. Goldbeter, Biochemical Oscillations and Cellular Rhythms (Cambridge University Press, Cambridge, 1996); Dynamic Biological Networks, edited by R. M. Harris-Warrik, E. Marder, A. I. Selverton, and M. Moulins (MIT Press, Cambridge, 1992).

[28] An interesting example to think about is the wake-sleep cycle of a brain.

[29] P. Holmes, J. L. Lumley, and G. Berkooz, Turbulence, Coherent Structures, Dynamical Systems and Symmetry (Cambridge University Press, Cambridge, 1996). 